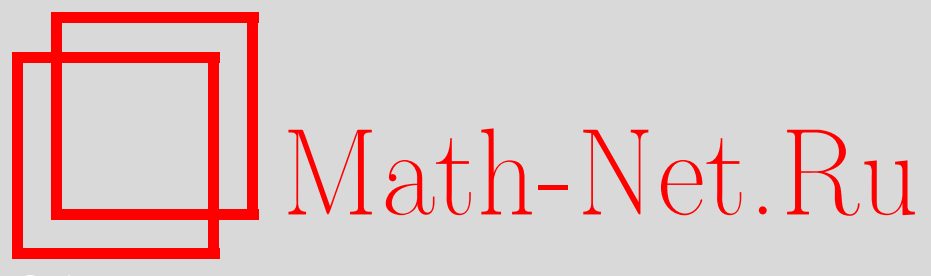

М. В. Коробков, Е. Ю. Панов, Об изэнтропических решениях квазилинейных уравнений первого порядка, $M a$ тем. сб., 2006, том 197, номер 5, 99-124

DOI: https://doi.org/10.4213/sm1560

Использование Общероссийского математического портала Math-Net.Ru подразумевает, что вы прочитали и согласны с пользовательским соглашением http://www . mathnet.ru/rus/agreement

Параметры загрузки:

IP : 54.164 .48 .24

26 апреля 2023 г., 16:11:58

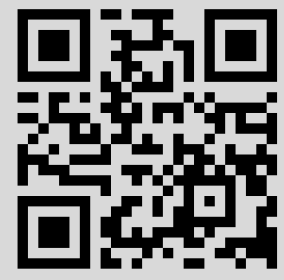




\author{
М. В. Коробков, Е. Ю. Панов
}

\title{
Об изэнтропических решениях квазилинейных уравнений первого порядка
}

Найдены условия существования нетривиальных изэнтропических решений квазилинейных законов сохранения. Даны приложения к проблеме функциональной зависимости частных производных гладкой функции двух переменных. В частности, найдены условия на функцию $\varphi$, необходимые для того, чтобы уравнение $\frac{\partial v}{\partial t}=\varphi\left(\frac{\partial v}{\partial x}\right)$ имело нетривиальные
$C^{1}$-гладкие решения.

Библиография: 13 названий.

\section{Введение}

Настоящая статья возникла в связи со следующим вопросом о функциональной зависимости частных производных гладкой функции двух переменных. Пусть $v=v(t, x)-C^{1}$-гладкая функция в области $\Omega \subset \mathbb{R}^{2}$, отличная от линейной, и

$$
v_{t}=\varphi\left(v_{x}\right) \quad \text { в } \Omega,
$$

где $\varphi=\varphi(u)$ - непрерывная функция из $\mathbb{R}$ в $\mathbb{R}$. Спрашивается, что можно сказать о свойствах $\varphi(u)$ ? Производные $v_{t}, v_{x}$ лишь непрерывны, и, как может показаться сначала, соотношение (1) не может дать ничего большего, чем естественное свойство непрерывности $\varphi(u)$. Оказалось, что функция $\varphi(u)$ должна удовлетворять следующему достаточно жесткому свойству:

(*) найдется замкнутое относителъно интервала $(a, b)=\operatorname{Int} v_{x}(\Omega)$ множество $F$ нулевой меры такое, что $\varphi(u)$ удовлетворяет условию Липшица локально на $U=(a, b) \backslash F$ и производная $\varphi^{\prime}(u)$ имеет локально ограниченную вариацию на $U$

(см. теорему 7 ниже). В частности, $\varphi(u)$ дважды (!) дифференцируема для почти всех $u \in(a, b)$. Здесь вторая производная $\varphi^{\prime \prime}(u)$ понимается в смысле обычного предела разностного отношения

$$
\lim _{s \rightarrow u} \frac{\varphi^{\prime}(s)-\varphi^{\prime}(u)}{s-u},
$$

Первый автор поддержан Российским фондом фундаментальных исследований (гранты №oo 02-01-01009, 05-01-00482), Программой Президента РФ поддержки молодых российский ученых (грант № MK-3778.2004.1) и Программой поддержки ведущих научных школ РФ (грант № НШ-311.2003.1). Второй автор поддержан Российским фондом фундаментальных исследований (грант № 03-01-00444) и Программой “Университеты России” (грант № УР.04.01.184).

(C) М. В. Коровков, Е. Ю. ПАнов, 2006 
но рассматриваемого не на всем интервале $(a, b)$ (как в классическом анализе), а на множестве существования первой производной $\varphi^{\prime}(s)$, которое имеет полную меру Лебега.

Для решения указанной проблемы оказались полезными результаты теории изэнтропических решений квазилинейных уравнений первого порядка, введенных в диссертации [1]. Эти результаты излагаются ниже в $\S \S 1,2$ и по мнению авторов представляют также и самостоятельный интерес.

Напомним, прежде всего, некоторые понятия теории обобщенных энтропийных решений задачи Коши для квазилинейного уравнения первого порядка

$$
\begin{gathered}
u_{t}+\operatorname{div}_{x} \varphi(u)=0, \\
\varphi=\left(\varphi_{1}, \ldots, \varphi_{n}\right), \quad u=u(t, x), \quad(t, x) \in \Pi_{T}=(0, T) \times \mathbb{R}^{n}, \quad 0<T \leqslant+\infty,
\end{gathered}
$$

с начальным условием

$$
u(0, x)=u_{0}(x) \in L^{\infty}\left(\mathbb{R}^{n}\right)
$$

Предполагается, что вектор потока $\varphi(u)$ лишь непрерывен: $\varphi \in C\left(\mathbb{R}, \mathbb{R}^{n}\right)$.

ОПРЕДЕЛЕНИЕ 1 (С.Н. Кружков [2], [3]). Ограниченная измеримая функция $u=u(t, x) \in L^{\infty}\left(\Pi_{T}\right)$ называется обобщенным энтропийным решением (коротко - о.э.р.) задачи Коши (2), (3), если

a) $\forall k \in \mathbb{R}$

$$
|u-k|_{t}+\operatorname{div}_{x}[\operatorname{sign}(u-k)(\varphi(u)-\varphi(k))] \leqslant 0
$$

в смысле распределений на $\Pi_{T}\left(\right.$ в $\left.\mathscr{D}^{\prime}\left(\Pi_{T}\right)\right)$;

b) $\operatorname{ess} \lim _{t \rightarrow 0+} u(t, \cdot)=u_{0}$ в $L_{\mathrm{loc}}^{1}\left(\mathbb{R}^{n}\right)$, т.е. существует множество $\mathscr{E} \subset(0, T)$ полной меры Лебега такое, что $u(t, \cdot) \rightarrow u_{0}$ в $L_{\text {loc }}^{1}\left(\mathbb{R}^{n}\right)$ при $t \rightarrow 0+, t \in \mathscr{E}$.

Условие (4) означает, что для любой пробной функции $f=f(t, x) \in C_{0}^{\infty}\left(\Pi_{T}\right)$, $f \geqslant 0$,

$$
\int_{\Pi_{T}}\left\{|u-k| f_{t}+\operatorname{sign}(u-k)\left(\varphi(u)-\varphi(k), \nabla_{x} f\right)\right\} d t d x \geqslant 0 .
$$

Здесь и ниже $(\cdot, \cdot)$ означает скалярное умножение в $\mathbb{R}^{n}$. Мы будем также использовать обозначение $|\cdot|$ для евклидовой нормы конечномерного вектора.

Положив в (4) $k= \pm\|u\|_{\infty}$, легко вывести, что $u_{t}+\operatorname{div}_{x} \varphi(u)=0$ в $\mathscr{D}^{\prime}\left(\Pi_{T}\right)$ и $u(t, x)$ удовлетворяет уравнению $(2)$ в смысле распределений, т.е. действительно является обобщенным решением (о.p.).

Как показано в [4], условия a), b) могут быть описаны одним интегральным неравенством, а именно (см. [4; предложение 1]) $u=u(t, x)-$ o.э.р. задачи $(2),(3)$ тогда и только тогда, когда для всех $k \in \mathbb{R}$ и для любой $f=f(t, x) \in$ $C_{0}^{\infty}\left([0, T) \times \mathbb{R}^{n}\right), f \geqslant 0$,

$$
\begin{gathered}
\int_{\Pi_{T}}\left\{|u-k| f_{t}+\operatorname{sign}(u-k)\left(\varphi(u)-\varphi(k), \nabla_{x} f\right)\right\} d t d x \\
+\int_{\mathbb{R}^{n}}\left|u_{0}(x)-k\right| f(0, x) d x \geqslant 0 .
\end{gathered}
$$

Известно (см. [1]-[7]), что о.э.р. задачи (2), (3) всегда существует, но в рассматриваемом случае, когда вектор потока лишь непрерывен, может быть не 
единственным, если число пространственных переменных $n>1$. Соответствующие примеры были впервые построены в работах [1], [5], [6]. Там же найдены некоторые достаточные условия единственности о.э.р. (из которых, в частности, следует, что о.э.р. всегда единственно при $n=1)$. Заметим также, что для непрерывного по Липшицу потока единственность о.э.р. хорошо известна (см. [2], [3]).

Мы рассматриваем здесь лишь ограниченные о.э.р. (в отличие от изэнтропических решений ниже), поскольку в более широком классе локально ограниченных о.э.р. постановка задачи Коши оказывается некорректной (см., например, [8]).

\section{§1. Изэнтропические решения}

Пусть $\Omega \subset \mathbb{R}^{n+1}$ - открытое связное подмножество (область).

ОПРЕДЕЛЕНИЕ 2 (см. [1]). Функция $u(t, x) \in L_{\mathrm{loc}}^{\infty}(\Omega)$ называется изэнтропическим решением (далее и.p.) уравнения (2) в области $\Omega$, если $\forall k \in \mathbb{R}$

$$
|u-k|_{t}+\operatorname{div}_{x}[\operatorname{sign}(u-k)(\varphi(u)-\varphi(k))]=0 \quad \text { в } \mathscr{D}^{\prime}(\Omega),
$$

т.е. энтропийное условие (4) выполняется в $\mathscr{D}^{\prime}(\Omega)$ со знаком равенства.

ЗАмЕЧАНИЕ 1. Из определения и.р. легко следует, что $u=u(t, x)$ является и.р. уравнения $(2)$ в области $\Omega$ тогда и только тогда, когда $-u-$ и.р. в $\Omega$ уравнения $u_{t}-\operatorname{div}_{x} \varphi(-u)=0$.

Отметим, что в [1] рассмотрены более общие изэнтропические мерозначные решения.

Условие (6) может быть переписано в более удобной форме. Справедливо

ПРЕДЛОЖЕНИЕ 1. Условие (6) эквивалентно любому из следующих условий:

1) $\forall k \in \mathbb{R} \max (u, k)_{t}+\operatorname{div}_{x} \varphi(\max (u, k))=0 \quad$ в $\mathscr{D}^{\prime}(\Omega)$

2) $\forall k \in \mathbb{R} \min (u, k)_{t}+\operatorname{div}_{x} \varphi(\min (u, k))=0 \quad$ в $\mathscr{D}^{\prime}(\Omega)$.

ДокАЗАТЕЛЬство. Заметим сначала, что

$$
\begin{aligned}
2 \max (u, k) & =|u-k|+(u+k), \\
2 \varphi(\max (u, k)) & =(\varphi(u)-\varphi(k)) \operatorname{sign}(u-k)+(\varphi(u)+\varphi(k)) ; \\
2 \min (u, k) & =-|u-k|+(u+k), \\
2 \varphi(\min (u, k)) & =-(\varphi(u)-\varphi(k)) \operatorname{sign}(u-k)+(\varphi(u)+\varphi(k)) .
\end{aligned}
$$

Далее, равенство

$$
u_{t}+\operatorname{div}_{x} \varphi(u)=0 \quad \text { в } \mathscr{D}^{\prime}(\Omega)
$$

является следствием как условия (6), так и любого из условий 1), 2) (при $k \rightarrow \pm \infty)$. Это вместе с равенствами (7), (8) доказывает эквивалентность условия (6) и условий 1), 2). Предложение доказано.

Предложение 1 является частным случаем следующего результата.

Теорема 1. Если $u_{1}=u_{1}(t, x), u_{2}=u_{2}(t, x)$ - и.p. уравнения (2) в области $\Omega$, то функиии $\max \left(u_{1}, u_{2}\right), \min \left(u_{1}, u_{2}\right)$ такље являются и.р. этого уравнения в $\Omega$. 
ДокАЗАТЕЛЬСтво. По условию 1) из предложения 1 для любого $k \in \mathbb{R}$

$$
\max (u, k)_{t}+\operatorname{div}_{x} \varphi(\max (u, k))=0 \quad \text { в } \quad \mathscr{D}^{\prime}(\Omega) .
$$

Применим метод “удвоения переменных", развитый С.Н. Кружковым в работах [2], [3]. Рассмотрим интегральное равенство, соответствующее (9) для $u=u_{1}(t, x)$ с пробной функцией $f(t, x ; \tau, y) \in C_{0}^{\infty}(\Omega \times \Omega)$ и $k=\max \left(u_{2}(\tau, y), c\right)$, $c \in \mathbb{R}$. Интегрируя это равенство на $\Omega(\tau, y)$, мы получаем, что

$$
v_{t}+\operatorname{div}_{x} \varphi(v)=0 \quad \text { в } \mathscr{D}^{\prime}(\Omega \times \Omega),
$$

$v=v(t, x ; \tau, y)=\max \left(u_{1}(t, x), u_{2}(\tau, y), c\right)$. Меняя ролями функции $u_{1}$ и $u_{2}$ и переменные $(t, x)$ и $(\tau, y)$, выводим аналогичное равенство:

$$
v_{\tau}+\operatorname{div}_{y} \varphi(v)=0 \quad \text { в } \mathscr{D}^{\prime}(\Omega \times \Omega) .
$$

Складывая (10) и (11), получаем, что

$$
\left(\frac{\partial}{\partial t}+\frac{\partial}{\partial \tau}\right) v+\left(\operatorname{div}_{x}+\operatorname{div}_{y}\right) \varphi(v)=0 \quad \text { в } \mathscr{D}^{\prime}(\Omega \times \Omega) .
$$

Выберем функцию

$$
\beta(t, x) \in C_{0}^{\infty}\left(\mathbb{R}^{n+1}\right), \quad \beta(t, x) \geqslant 0, \quad \int \beta(t, x) d t d x=1,
$$

и положим $\delta_{\nu}(t, x)=\nu^{n+1} \beta(\nu t, \nu x)$ при $\nu \in \mathbb{N}$. Понятно, что последовательность $\delta_{\nu}(t, x)$ сходится к $\delta$-мере Дирака в $\mathscr{D}^{\prime}\left(\mathbb{R}^{n+1}\right)$ при $\nu \rightarrow \infty$.

Пусть $f(t, x) \in C_{0}^{\infty}(\Omega), \nu \in \mathbb{N}$. Положим $g(t, x ; \tau, y)=f(t, x) \delta_{\nu}(t-\tau, x-y)$. Очевидно, при достаточно больших $\nu \in \mathbb{N}$ функция $g(t, x ; \tau, y) \in C_{0}^{\infty}(\Omega \times \Omega)$ и $\operatorname{supp} g \subset K \times K$, где $K \subset \Omega$ - некоторый компакт. Применяя равенство (12) к пробной функции $g$, получаем интегральное равенство

$$
\int_{\Omega(t, x)} \int_{\Omega(\tau, y)}\left\{v f_{t}+\left(\varphi(v), \nabla_{x} f\right)\right\} \delta_{\nu}(t-\tau, x-y) d \tau d y d t d x=0 .
$$

Пусть $w=w(t, x)=v(t, x ; t, x)=\max \left(u_{1}(t, x), u_{2}(t, x), c\right)$,

$$
\begin{aligned}
I_{\nu}(t, x) & =\int_{\Omega(\tau, y)}\left\{v f_{t}+\left(\varphi(v), \nabla_{x} f\right)\right\} \delta_{\nu}(t-\tau, x-y) d \tau d y, \\
I(t, x) & =w(t, x) f_{t}(t, x)+\left(\varphi(w(t, x)), \nabla_{x} f(t, x)\right) \\
& =\int_{\Omega(\tau, y)}\left\{w f_{t}+\left(\varphi(w), \nabla_{x} f\right)\right\} \delta_{\nu}(t-\tau, x-y) d \tau d y .
\end{aligned}
$$

Учитывая, что $|v(t, x ; \tau, y)-w(t, x)| \leqslant\left|u_{2}(\tau, y)-u_{2}(t, x)\right|$ и для п.в. $(t, x, \tau, y) \in$ $K \times K$

$$
|\varphi(v(t, x ; \tau, y))-\varphi(w(t, x))| \leqslant \omega\left(\left|u_{2}(\tau, y)-u_{2}(t, x)\right|\right),
$$

где $\omega(r)=\sup \{|\varphi(u)-\varphi(v)|: u, v \in[-M, M],|u-v|<r\}-$ модуль непрерывности вектора $\varphi(u)$ на отрезке $[-M, M]$ с $M=\max \left(\left\|u_{1}\right\|_{L^{\infty}(K)},\left\|u_{2}\right\|_{L^{\infty}(K)}\right)$, получаем оценку

$$
\begin{aligned}
\left|I_{\nu}(t, x)-I(t, x)\right| \leqslant C_{f} & \int_{\Omega}\left[\left|u_{2}(\tau, y)-u_{2}(t, x)\right|+\omega\left(\left|u_{2}(\tau, y)-u_{2}(t, x)\right|\right)\right] \\
& \times \delta_{\nu}(t-\tau, x-y) d \tau d y, \quad C_{f}=\mathrm{const},
\end{aligned}
$$


из которой следует, что $I_{\nu}(t, x) \rightarrow I(t, x)$ при $\nu \rightarrow \infty$ на множестве полной меры точек Лебега функции $u_{2}(t, x)$. Ввиду ограниченности $u_{1}, u_{2}$ на компакте $K$ также $\left\|I_{\nu}\right\|_{\infty} \leqslant$ const. По теореме Лебега о предельном переходе под знаком интеграла тогда справедливо соотношение

$$
\int_{\Omega} I_{\nu}(t, x) d t d x \underset{\nu \rightarrow \infty}{\longrightarrow} \int_{\Omega} I(t, x) d t d x,
$$

и так как $\int_{\Omega} I_{\nu}(t, x) d t d x=0$ ввиду (13), то $\int_{\Omega} I(t, x) d t d x=0$ или, расписав,

$$
\int_{\Omega}\left[w(t, x) f_{t}(t, x)+\left(\varphi(w(t, x)), \nabla_{x} f(t, x)\right)\right] d t d x=0 .
$$

Последнее ввиду произвольности $f \in C_{0}^{\infty}(\Omega)$ означает, что $w_{t}+\operatorname{div}_{x} \varphi(w)=0$ в $\mathscr{D}^{\prime}(\Omega)$, т.е. при $p=p(t, x)=\max \left(u_{1}, u_{2}\right)$

$$
\max (p, c)_{t}+\operatorname{div}_{x} \varphi(\max (p, c))=0 \quad \text { в } \quad \mathscr{D}^{\prime}(\Omega) \quad \forall c \in \mathbb{R} .
$$

По предложению $1 p=\max \left(u_{1}, u_{2}\right)$ - и.р. уравнения (2) в $\Omega$. Наконец, с учетом равенства $\min \left(u_{1}, u_{2}\right)=-\max \left(-u_{1},-u_{2}\right)$ и замечания $1 \min \left(u_{1}, u_{2}\right)$ также является и.р. уравнения (2). Теорема доказана.

Ниже, при доказательстве теоремы 4, нам понадобится следующее

ПрЕДЛОЖЕНИЕ 2. Пусть $u(t, x) \in L_{\mathrm{loc}}^{\infty}\left(\Pi_{T}\right)-u . p$. уравнения $(2),-\infty \leqslant m=$ ess inf $u(t, x)$, ess $\sup u(t, x)=M \leqslant+\infty$. Тогда существует множество полной меры $E \subset(0, T)$ такое, что на этом множестве отображение $t \mapsto u(t, \cdot)$ непрерывно в топологии $L_{\mathrm{loc}}^{1}\left(\mathbb{R}^{n}\right) u \forall t \in E$

$$
\underset{x \in \mathbb{R}^{n}}{\operatorname{essinf}} u(t, x)=m, \quad \quad \underset{x \in \mathbb{R}^{n}}{\operatorname{ess} \sup } u(t, x)=M .
$$

ДоказАтельство. Рассмотрим сначала случай, когда и.р. $u(t, x)$ ограничено: $u(t, x) \in L^{\infty}\left(\Pi_{T}\right)$. Выберем, как в доказательстве теоремы 1 , функцию $\beta(t) \in C_{0}^{\infty}(\mathbb{R})$ так, что

$$
\operatorname{supp} \beta \subset[0,1], \quad \beta(t) \geqslant 0, \quad \int \beta(t) d t=1,
$$

и положим при $\nu \in \mathbb{N}$

$$
\delta_{\nu}(t)=\nu \beta(\nu t), \quad \theta_{\nu}(t)=\int_{0}^{t} \delta_{\nu}(s) d s,
$$

так что $\theta_{\nu}^{\prime}(t)=\delta_{\nu}(t)$. Ясно, что последовательность $\delta_{\nu}(t)$ сходится при $\nu \rightarrow \infty$ к $\delta$-мере Дирака в $\mathscr{D}^{\prime}(\mathbb{R})$, а последовательность $\theta_{\nu}(t)$ поточечно сходится к функции Хевисайда

$$
\theta(t)= \begin{cases}0, & t \leqslant 0 \\ 1, & t>0\end{cases}
$$


Пусть $t_{0} \in(0, T), f=f(t, x) \in C_{0}^{\infty}\left(\Pi_{T}\right)$. Применяя соотношение $(6)$ к пробной функции $f \theta_{\nu}\left(t-t_{0}\right)$, после элементарных преобразований получаем, что $\forall k \in \mathbb{R}$

$$
\begin{aligned}
\int_{0}^{T} & \left(\int_{\mathbb{R}^{n}}|u(t, x)-k| f(t, x) d x\right) \delta_{\nu}\left(t-t_{0}\right) d t \\
& +\int_{\Pi_{T}}\left\{|u-k| f_{t}+\operatorname{sign}(u-k)\left(\varphi(u)-\varphi(k), \nabla_{x} f\right)\right\} \theta_{\nu}\left(t-t_{0}\right) d t d x=0 .
\end{aligned}
$$

Пусть $S \subset C_{0}^{\infty}\left(\Pi_{T}\right)$ - счетное всюду плотное (относительно стандартной локально выпуклой топологии $\left.C_{0}^{\infty}\left(\Pi_{T}\right)\right)$ множество и $E \subset(0, T)$ - множество общих точек Лебега функций

$$
t \mapsto \int_{\mathbb{R}^{n}}|u(t, x)-k| f(t, x) d x
$$

при $f \in S$ и рациональных $k \in \mathbb{Q}$. Учитывая, что множества $S$ и $\mathbb{Q}$ счетны, заключаем, что $E$ имеет полную меру Лебега на $(0, T)$. Для $t_{0} \in E$ из (14) в пределе при $\nu \rightarrow \infty$ следует соотношение

$$
\begin{aligned}
\int_{\mathbb{R}^{n}} \mid & u\left(t_{0}, x\right)-k \mid f\left(t_{0}, x\right) d x \\
& +\int_{\Pi_{T}}\left\{|u-k| f_{t}+\operatorname{sign}(u-k)\left(\varphi(u)-\varphi(k), \nabla_{x} f\right)\right\} \theta\left(t-t_{0}\right) d t d x=0 .
\end{aligned}
$$

Так как множества $S \subset C_{0}^{\infty}\left(\Pi_{T}\right)$ и $\mathbb{Q} \subset \mathbb{R}$ плотны, а интегралы в (15) непрерывно зависят от $f$ и $k$, то соотношение (15) справедливо для всех $f \in C_{0}^{\infty}\left(\Pi_{T}\right)$ и $k \in$ $\mathbb{R}$. В частности, $u(t, x)$ удовлетворяет условию $(5)$ с заменой $\Pi_{T}$ на $\left(t_{0}, T\right) \times \mathbb{R}^{n}$, a $u_{0}(x)$ - на $u\left(t_{0}, x\right)$. Поэтому $u(t, x)$ является о.э.р. задачи Коши в этом слое с начальной функцией $u\left(t_{0}, x\right)$ и по начальному условию b)

$$
\underset{t \rightarrow t_{0}+}{\operatorname{ess}} \lim _{t} u(t, \cdot)=u\left(t_{0}, \cdot\right) \quad \text { в } \quad L_{\text {loc }}^{1}\left(\mathbb{R}^{n}\right) .
$$

Далее, поменяем направление "времени" и рассмотрим функцию $u(T-t,-x)$. Легко видеть, что эта функция также является и.р. уравнения (2) и, значит, для нее справедливо соотношение вида (16), которое означает, что при $t_{0} \in E$

$$
\underset{t \rightarrow t_{0}-}{\operatorname{ess}} \lim u(t, \cdot)=u\left(t_{0}, \cdot\right) \quad \text { в } \quad L_{\mathrm{loc}}^{1}\left(\mathbb{R}^{n}\right) .
$$

Итак, $\forall t_{0} \in E$

$$
\underset{t \rightarrow t_{0}}{\operatorname{ess}} \lim u(t, \cdot)=u\left(t_{0}, \cdot\right) \quad \text { в } \quad L_{\mathrm{loc}}^{1}\left(\mathbb{R}^{n}\right) .
$$

Из (17) легко следует, что $\forall t_{0} \in E$

$$
\lim _{t \rightarrow t_{0}, t \in E} u(t, \cdot)=u\left(t_{0}, \cdot\right) \quad \text { в } L_{\mathrm{loc}}^{1}\left(\mathbb{R}^{n}\right)
$$

и отображение $t \mapsto u(t, \cdot)$ непрерывно в топологии $L_{\mathrm{loc}}^{1}\left(\mathbb{R}^{n}\right)$. Далее, при $t_{0} \in E$ функции $u_{+}(t, x)=u\left(t+t_{0}, x\right)$ и $u_{-}(t, x)=u\left(t_{0}-t,-x\right)$ являются ограниченными и.p. (а значит, и о.э.p.) задачи (2), (3) в слоях $\Pi_{T-t_{0}}$ и $\Pi_{t_{0}}$ соответственно с начальными данными $u\left(t_{0}, x\right)$ и $u\left(t_{0},-x\right)$. Обозначим

$$
\begin{aligned}
& m_{0}=\underset{x \in \mathbb{R}^{n}}{\operatorname{essinf}} u\left(t_{0}, x\right)=\underset{x \in \mathbb{R}^{n}}{\operatorname{essinf}} u\left(t_{0},-x\right), \\
& M_{0}=\underset{x \in \mathbb{R}^{n}}{\operatorname{ess} \sup } u\left(t_{0}, x\right)=\underset{x \in \mathbb{R}^{n}}{\operatorname{ess} \sup _{0}} u\left(t_{0},-x\right) .
\end{aligned}
$$


Тогда по принципу максимума (см. [4], [7]) $m_{0} \leqslant u_{+}(t, x) \leqslant M_{0}$ и $m_{0} \leqslant$ $u_{-}(t, x) \leqslant M_{0}$ П.в. в $\Pi_{T-t_{0}}$ и $\Pi_{t_{0}}$ соответственно, т.е. $m_{0} \leqslant u(t, x) \leqslant M_{0}$ п.в. в $\Pi_{T}$. Поэтому

$$
m_{0} \leqslant m=\operatorname{essinf} u(t, x) \leqslant \operatorname{ess} \sup u(t, x)=M \leqslant M_{0}
$$

и так как обратные неравенства $m \leqslant m_{0}, M_{0} \leqslant M$ очевидны (с учетом непрерывности $u(t, \cdot)$ в $L_{\text {loc }}^{1}\left(\mathbb{R}^{n}\right)$ при $\left.t \in E\right)$, то $\forall t_{0} \in E$

$$
m_{0}=\underset{x \in \mathbb{R}^{n}}{\operatorname{essinf}} u(t, x)=m, \quad M_{0}=\underset{x \in \mathbb{R}^{n}}{\operatorname{essip}} u(t, x)=M,
$$

что и требовалось доказать. Предложение в случае ограниченного $u(t, x)$ доказано.

В общем случае $u(t, x) \in L_{\mathrm{loc}}^{\infty}\left(\Pi_{T}\right)$ рассмотрим "срезки"

$$
u_{r}(t, x)=\max (-r, \min (u(t, x), r)), \quad r \in \mathbb{N} .
$$

По теореме 1 функции $u_{r}(t, x)$ являются и.р. уравнения $(2)$, и поскольку эти и.р. ограничены, то для них утверждения предложения 2 уже доказаны и выполнены на множествах полной меры $E_{r}$. Для завершения доказательства остается лишь положить $E=\bigcap_{r \in \mathbb{N}} E_{r}$ и устремить $r$ к бесконечности. Предложение полностью доказано.

В линейном случае, когда $\varphi(u)=(c, u), c \in \mathbb{R}^{n}$, любое обобщенное решение уравнения (2) является изэнтропическим. Это легко следует из условия постоянства обобщенного решения на характеристиках $x-c t=$ const. Для нелинейного уравнения (2) существование нетривиального и.р. налагает довольно жесткие ограничения на характер нелинейности (см. §2 ниже).

В случае, когда функции потока непрерывны по Липшицу, и.р. задачи Коши для уравнения (2) допускают простое описание. Точнее, предположим, что $u_{0}(x) \in[a, b]$ п.в. в открытом шаре $V_{r}=\left\{x \in \mathbb{R}^{n}:|x|<r\right\}, 0<r \leqslant \infty$, и

$$
|\varphi(u)-\varphi(v)| \leqslant N|u-v| \quad \forall u, v \in[a, b],
$$

$\Omega=\Omega_{r}-$ конус $\left\{(t, x) \in \Pi_{T}:|x|+N t<r\right\}$ (заметим, что $\Omega=\Pi_{T}$ при $r=\infty$ ). Поскольку вектор потока удовлетворяет условию Липшица (18), вектор обобщенных производных $\varphi^{\prime}(u)$ лежит в $L^{\infty}([a, b])$ и $\left|\varphi^{\prime}(u)\right| \leqslant N$. Определим при $(t, x) \in \Omega$ функции

$$
\begin{aligned}
& u^{+}(t, x)=\inf \left\{u \in[a, b]: v>u_{0}\left(x-\varphi^{\prime}(v) t\right) \text { для п.в. } v \in(u, b)\right\}, \\
& u^{-}(t, x)=\sup \left\{u \in[a, b]: v<u_{0}\left(x-\varphi^{\prime}(v) t\right) \text { для п.в. } v \in(a, u)\right\} .
\end{aligned}
$$

Заметим, что $x-\varphi^{\prime}(v) t \in V_{r}$ при $(t, x) \in \Omega$ ввиду оценки $\left|\varphi^{\prime}(v)\right| \leqslant N$. Пусть функция $u(t, x) \in L^{\infty}(\Omega)$ такова, что $u(t, x) \in[a, b]$ п.в. на $\Omega$ и $u(0, x)=u_{0}(x)$ в смысле соотношения

$$
\underset{t \rightarrow 0}{\operatorname{ess} \lim _{|x|<r-N t}} \int_{\left|u(t, x)-u_{0}(x)\right| d x=0 .}
$$

Тогда справедлива следующая теорема. 
ТЕОРЕма 2. Функиия $и(t, x)$ является и.р. уравнения (2) в области $\Omega$ тогда и только тогда, когда $u^{+}(t, x)=u^{-}(t, x)=u(t, x)$ n.в. в $\Omega$.

ДокАЗАТЕЛЬСтво. Положим при $(t, x, v) \in \Omega \times(a, b)$

$$
U(t, x, v)=\theta(v-u(t, x)), \quad U_{0}(x, v)=\theta\left(v-u_{0}(x)\right), \quad \theta(v)= \begin{cases}1, & v>0 \\ \frac{1}{2}, & v=0 \\ 0, & v<0\end{cases}
$$

Легко проверяется, что при $k \in \mathbb{R}, u=u(t, x) \in[a, b]$

$$
\begin{aligned}
|u-k|=-\int_{a}^{b} \operatorname{sign}(v-k) U(t, x, v) d v+|b-k| & \\
\operatorname{sign}(u-k)(\varphi(u)-\varphi(k))=- & \int_{a}^{b} \operatorname{sign}(v-k) \varphi^{\prime}(v) U(t, x, v) d v \\
& +\operatorname{sign}(b-k)(\varphi(b)-\varphi(k)) .
\end{aligned}
$$

Поэтому при $f=f(t, x) \in C_{0}^{\infty}(\Omega)$

$$
\begin{aligned}
\int_{\Omega}\left[|u-k| f_{t}+\operatorname{sign}(u-k)\left(\varphi(u)-\varphi(k), \nabla_{x} f\right)\right] d t d x \\
=-\int_{a}^{b} \operatorname{sign}(v-k)\left(\int_{\Omega} U(t, x, v)\left[f_{t}+\left(\varphi^{\prime}(v), \nabla_{x} f\right)\right] d t d x\right) d v .
\end{aligned}
$$

Мы учли, что величины $c_{0}=|b-k|$ и $c=\operatorname{sign}(b-k)(\varphi(b)-\varphi(k)) \in \mathbb{R}^{n}$ не зависят от $(t, x)$ и поэтому

$$
\int_{\Omega}\left[c_{0} f_{t}+\left(c, \nabla_{x} f\right)\right] d t d x=0 .
$$

Кроме того, из (19) следует, что

$$
\underset{t \rightarrow 0}{\operatorname{ess} \lim _{a}} \int_{a}^{b} \int_{|x|<r-N t}\left|U(t, x, v)-U_{0}(x, v)\right| d x d v=0 .
$$

При

$$
I_{f}(v)=\int_{\Omega} U(t, x, v)\left[f_{t}+\left(\varphi^{\prime}(v), \nabla_{x} f\right)\right] d t d x
$$

соотношение (20) показывает, что $u(t, x)$ является и.р. уравнения $(2)$ в $\Omega$ тогда и только тогда, когда $\forall k \in \mathbb{R}, f \in C_{0}^{\infty}(\Omega)$

$$
\int_{a}^{b} \operatorname{sign}(v-k) I_{f}(v) d v=0
$$

т.е. $I_{f}(v)=0$ п.в. на $(a, b)$. Выберем счетное всюду плотное (относительно стандартной локально выпуклой топологии на $\left.C_{0}^{\infty}(\Omega)\right)$ подмножество $S \subset C_{0}^{\infty}(\Omega)$. Тогда множество $E=\left\{v \in(a, b): I_{f}(v)=0 \forall f \in S\right\}$ имеет полную меру. Поскольку $S$ плотно в $C_{0}^{\infty}(\Omega)$ и при фиксированном $v \in E$ соответствие $f \rightarrow I_{f}(v)$ непрерывно на $C_{0}^{\infty}(\Omega)$, то $I_{f}(v)=0 \forall f \in C_{0}^{\infty}(\Omega)$, т.е. при $v \in E$

$$
U_{t}+\left(\varphi^{\prime}(v), \nabla_{x} U\right)=0
$$


в $\mathscr{D}^{\prime}(\Omega)$. Поэтому $U(t, x, v)$ - о.р. линейного уравнения $(22)$ и, значит, оно постоянно на характеристиках $x-\varphi^{\prime}(v) t=$ const. Последнее означает, что $U(t, x, v)=g\left(x-\varphi^{\prime}(v) t, v\right)$ для п.в. $(t, x) \in \Omega$, где $g(\cdot, v)$ - некоторая измеримая ограниченная функция на $V_{r}$. Далее, из предельного соотношения (21) следует, что $g(x, v)=U_{0}(x, v)$ п.в. на $V_{r} \times(a, b)$ и, значит,

$$
U(t, x, v)=U_{0}\left(x-\varphi^{\prime}(v) t, v\right)=\theta\left(v-u_{0}\left(x-\varphi^{\prime}(v) t\right)\right) \quad \text { п.в. на } \Omega \times(a, b) .
$$

Итак, требование, что $u(t, x)$ является и.р., эквивалентно выполнению равенства (23). Последнее означает, в свою очередь, что для п.в. $(t, x) \in \Omega$

$$
\begin{array}{lll}
v>u_{0}\left(x-\varphi^{\prime}(v) t\right) & \text { при п.в. } v>u(t, x), \\
v<u_{0}\left(x-\varphi^{\prime}(v) t\right) & \text { при п.в. } v<u(t, x),
\end{array}
$$

т.е. $u^{+}(t, x)=u^{-}(t, x)=u(t, x)$ п.в. в $\Omega$. Теорема доказана.

\section{§ 2. Случай одной пространственной переменной}

Рассмотрим случай, когда размерность $n=1$ и уравнение $(2)$ имеет вид

$$
u_{t}+\varphi(u)_{x}=0
$$

$u=u(t, x),(t, x) \in \Omega \subset \mathbb{R}^{2}$.

В этом случае справедливо следующее достаточное условие изэнтропичности решения.

Теорема 3. Пусть $u(t, x) \in C(\Omega)$ - непрерывное о.р. уравнения (24) в области $\Omega \subset \mathbb{R}^{2}$. Тогда $и(t, x)$ - изэнтропическое решение.

Для доказательства теоремы 3 нам понадобится следующая простая техническая

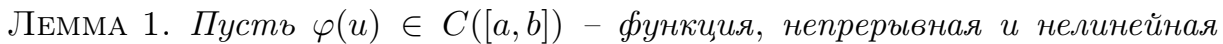
на отрезке $[a, b]$. Тогда найдется $c \in \mathbb{R} u u_{0} \in(a, b)$ - точка строгого экстремума $\varphi(u)-$ си на $[a, b]$.

ДокАЗАТЕЛЬСтво. Пусть $h(c, u)=\varphi(u)-c u, c^{*}=(\varphi(b)-\varphi(a)) /(b-a)$. Тогда по условию нелинейности $h\left(c^{*}, u\right)$ отлично от константы $h\left(c^{*}, a\right)=h\left(c^{*}, b\right)$. Пусть для определенности $\exists u \in(a, b): h\left(c^{*}, u\right)<h\left(c^{*}, a\right)=h\left(c^{*}, b\right)$. По непрерывности найдется $\delta>0$ такое, что

$$
\min _{u \in[a, b]} h(c, u)<\min (h(c, a), h(c, b)) \quad \forall c \in\left(c^{*}-\delta, c^{*}+\delta\right) .
$$

Заметим далее, что условие $h\left(c, u_{0}\right)=\min _{u \in[a, b]} h(c, u)$ переписывается в виде $\varphi(u) \geqslant l(u)=c\left(u-u_{0}\right)+\varphi\left(u_{0}\right) \forall u \in[a, b]$, откуда следует, что $\varphi^{*}(u) \geqslant c\left(u-u_{0}\right)+$ $\varphi\left(u_{0}\right) \geqslant c\left(u-u_{0}\right)+\varphi^{*}\left(u_{0}\right) \forall u \in[a, b]$, где $\varphi^{*}(u)$ - выпуклая оболочка функции $\varphi(u)$ на отрезке $[a, b]$. В частности, при $u=u_{0}$ получаем, что $\varphi\left(u_{0}\right)=\varphi^{*}\left(u_{0}\right)$. Итак, мы видим, что из условия

$$
\varphi\left(u_{0}\right)-c u_{0}=\min _{u \in[a, b]}(\varphi(u)-c u)
$$


следует, что

$$
\varphi^{*}\left(u_{0}\right)-c u_{0}=\min _{u \in[a, b]}\left(\varphi^{*}(u)-c u\right) .
$$

Поэтому если $u_{1}, u_{2}$ - точки минимума функции $\varphi(u)-c u, u_{1}<u_{2}$, то эти точки являются также точками минимума $\varphi^{*}(u)-c u$, а поскольку функция $\varphi^{*}(u)$ выпукла, то множество точек минимума функции $\varphi^{*}(u)-c u$ содержит отрезок $\left[u_{1}, u_{2}\right]$. Это означает, что функция $\varphi^{*}(u)$ линейна на $\left(u_{1}, u_{2}\right)$ с угловым коэффициентом $c$. Ясно, что указанные интервалы $\left(u_{1}, u_{2}\right)$ при разных значениях $c$ не пересекаются и, следовательно, их множество не более чем счетно. Значит, то же верно для соответствующего множества угловых коэффициентов $S$, состоящего из значений $c \in \mathbb{R}$, для которых функция $\varphi(u)-c u$ достигает минимума для более чем одного значения аргумента. Следовательно, найдется значение $c \in\left(c^{*}-\delta, c^{*}+\delta\right) \backslash S$ и этому значению соответствует единственная точка $u_{0} \in[a, b]$, для которой выполнено (26). При этом, как следует из (25), $u_{0} \neq a, u_{0} \neq b$ и $u_{0} \in(a, b)$. По построению $\varphi(u)-c u>\varphi\left(u_{0}\right)-c u_{0} \forall u \in[a, b]$, $u \neq u_{0}$, и $u_{0}$ - точка строгого минимума функции $\varphi(u)-c u$. Лемма доказана.

ДОКАЗАТЕЛЬСТВО ТЕОРЕМЫ 3. По определению изэнтропичности и предложению 1 требуется доказать, что $\forall k \in \mathbb{R}$ в $\mathscr{D}^{\prime}(\Omega)$

$$
\max (u, k)_{t}+\varphi(\max (u, k))_{x}=0 .
$$

Очевидно, достаточно проверить выполнение соотношения (27) для всюду плотного множества значений $k$. Рассмотрим два удобных для работы множества, объединение которых всюду плотно:

- $K_{1}$ состоит из точек $k$, в окрестности которых функция $\varphi(u)$ линейна, т.е. $\exists \delta>0, c \in \mathbb{R}: \varphi(u) \equiv \varphi(k)+c(u-k)$ при $|u-k|<\delta$;

- $K_{2}$ состоит из точек $k$, для которых найдутся значения $c \in \mathbb{R}$ и $\delta>0$ такие, что $k$ является точкой строгого экстремума функции $\varphi(u)-c u$ на интервале $|u-k|<\delta$.

Как непосредственно следует из леммы 1 , объединение $K_{1} \cup K_{2}$ всюду плотно B $\mathbb{R}$.

Зафиксируем $k$, принадлежащее одному из указанных множеств. Добавляя к функциям $u$ и $\varphi(u)$ соответствующие константы, можем без потери общности считать, что

$$
k=\varphi(k)=0 .
$$

Далее, после линейной замены $x \rightarrow x-c t$ в уравнении (24) функция потока $\varphi(u)$ заменится на $\varphi(u)-c u$, при этом соответствующее отображение $u(t, x) \rightarrow u(t, x-c t)$, очевидно, сохраняет классы обобщенных и изэнтропических решений. Поэтому мы можем считать, что угловой коэффициент $c$, входящий в определение $k \in K_{1} \cup K_{2}$, равен нулю.

Достаточно проверить выполнение условия (27) локально, т.е. что для любой точки $w=\left(t_{0}, x_{0}\right) \in \Omega$ существует окрестность $U(w)$ такая, что равенство $(27)$ выполнено в $\mathscr{D}^{\prime}(U(w))$. Если $u(w) \neq 0$, то по непрерывности это свойство сохраняется и в достаточно малой окрестности $U=U(w)$ точки $w$. Тогда либо $\max (u, 0)=u$, либо $\max (u, 0)=0$ на $U$, и в любом из этих случаев $\max (u, k)_{t}+\varphi(\max (u, k))_{x}=0$ в $\mathscr{D}^{\prime}(U)$, т.е. условие $(27)$ выполнено в окрестности $U$. Остается исследовать случай $u(w)=0, w=\left(t_{0}, x_{0}\right) \in \Omega$. Ввиду 
локальности нашей задачи мы можем заменить область $\Omega$ на достаточно малую окрестность точки $w$ и считать, что $|u(t, x)|<\delta,|\varphi(u(t, x))|<\delta$ для всех $(t, x) \in \Omega$, где $\delta$ - параметр из определения множеств $K_{1}$ и $K_{2}$. Наконец, учитывая инвариантность классов обобщенных и изэнтропических решений относительно преобразований подобия $t^{\prime}=\lambda\left(t-t_{0}\right), x^{\prime}=\lambda\left(x-x_{0}\right), \lambda \neq 0$, будем отныне считать, что область $\Omega$ является квадратом, скажем $\Omega=(0,1)^{2}$ и $u(t, x) \in C(\bar{\Omega})$, $\bar{\Omega}=\mathrm{Cl} \Omega=[0,1]^{2}$.

Случай, соответствующий множеству $K_{1}$, совершенно тривиален, так как тогда $\varphi(u) \equiv$ const на множестве значений $u(t, x)$ (напомним, что $c=0$ ) и уравнение (24) имеет форму $u_{t}=0$.

Рассмотрим случай $k \in K_{2}$. Для определенности будем считать, что $k=0$ есть точка строгого локального максимума функции $\varphi(u)$, т.е. $\varphi(u)<\varphi(0)=0$ при $0<|u|<\delta$. Вследствие равенства (24) существует непрерывно дифференцируемая функция $v: \bar{\Omega} \rightarrow \mathbb{R}$ (потенциал), $v \in C^{1}(\bar{\Omega})$, такая, что $v_{x}(t, x)=$ $u(t, x), \quad v_{t}(t, x)=-\varphi(u(t, x))$ при $(t, x) \in \bar{\Omega}$. По условию

$$
v_{t}(t, x)=-\varphi(u(t, x)) \geqslant 0 \quad \text { на } \Omega
$$

и функция $v(t, x)$ не убывает по координате $t$.

Нам понадобится одно следствие из формулы коплощади (coarea formula, см., например, [9; теорема 3.2.11]).

Теорема (формула коплощади). Пусть $v: \Omega \rightarrow \mathbb{R}$ есть липшичева функиия в ограниченной области $\Omega \subset \mathbb{R}^{2}$, и пусть $B \subset \mathbb{R}^{2}$ - борелевское множество. Тогда для почти всех значений $y \in \mathbb{R}$ прообраз $v^{-1}(y)$ есть 1- $\sigma$-спрямляемое множество (т.е. множество $v^{-1}(y)$ можно покрыть счетным набором липшицевых кривых), причем длина $\mathscr{H}^{1}\left(v^{-1}(y)\right) \neq \infty$ (т.е. одномерная мера Хаусдорфа множества $v^{-1}(y)$ конечна) $u$

$$
\int_{B}|\nabla v(t, x)| d t d x=\int_{\mathbb{R}} \mathscr{H}^{1}\left(v^{-1}(y) \cap B\right) d y .
$$

В частности, для множества $S=\left\{(t, x) \in \Omega: v_{x}(t, x)=v_{t}(t, x)=0\right\}$ и для почти всех значений $y \in \mathbb{R}$ справедливо следующее утверждение: длина прообраза $v^{-1}(y)$ конечна, а длина пересечения $S \cap v^{-1}(y)$ равна нулю, т.е.

$$
\mathscr{H}^{1}\left(v^{-1}(y)\right)<\infty, \quad \mathscr{H}^{1}\left(v^{-1}(y) \cap S\right)=0 .
$$

Кроме того, как следует из классической теоремы Морса-Сарда (см., например, $\left[10\right.$; теорема 1.3]) и эквивалентности условий $v_{x}(t, x)=0$ и $v_{t}(t, x)=0$, почти все $y \in \mathbb{R}$ являются регулярными (в классическом смысле) значениями $v(t, x)$ на границе $\partial \Omega$, т.е. $v^{-1}(y)$ не содержит вершин квадрата $\bar{\Omega}$ и

$$
v_{x}(t, x) \neq 0, \quad v_{t}(t, x) \neq 0 \quad \forall(t, x) \in \partial \Omega \cap v^{-1}(y) .
$$

Значения $y$, для которых выполнены условия (29), (30), будем называть регулярными. Множество регулярных значений $у$ таких, что $v^{-1}(y) \neq \varnothing$, будем обозначать через $Y$. Из сформулированной теоремы о коплощади следует, что мера разности $v(\Omega) \backslash Y$ равна 0. Если $y \in Y$, то при фиксированном $x$ множество $\{t: v(t, x)=y\}$ не может содержать более одной точки. Действительно, если $v\left(t_{1}, x\right)=v\left(t_{2}, x\right)=y, t_{1}<t_{2}$, то в силу монотонности $v(t, x)$ по переменной $t \quad v(t, x)=y$ и $v_{t}(t, x)=-\varphi(u(t, x))=0 \forall t \in\left[t_{1}, t_{2}\right]$. Так как $\varphi(u)=0$ 
только при $u=0$, то также $v_{x}(t, x)=0 \forall t \in\left[t_{1}, t_{2}\right]$. Мы видим, что множество $S \cap v^{-1}(y)$ содержит отрезок $\left[t_{1}, t_{2}\right] \times\{x\}$ и $\mathscr{H}^{1}\left(v^{-1}(y) \cap S\right)>0$, что противоречит регулярности значения $y$.

Ясно, что множество $D_{y}$ тех $x$, для которых $\exists t(x): v(t(x), x)=y$, замкнуто как проекция компакта $v^{-1}(y) \subset \bar{\Omega}$ на ось $x$. Так как при $y \in Y$ отображение проекции инъективно на компакте $v^{-1}(y)$, оно является гомеоморфизмом компакта $v^{-1}(y)$ на $D_{y}$. Легко видеть, что для регулярного $y$ компоненты связности множества $D_{y}$ являются невырожденными отрезками. Действительно, пусть $x_{0} \in D_{y}$ и $t_{0}=t\left(x_{0}\right) \in[0,1]$ - единственное значение такое, что $v\left(t_{0}, x_{0}\right)=y$. Если $t_{0} \in(0,1)$, то с учетом монотонности $v\left(\cdot, x_{0}\right)$ $v\left(0, x_{0}\right)<y<v\left(1, x_{0}\right)$. По непрерывности $v(0, x)<y<v(1, x)$ для всех $x$ из достаточно малой окрестности $U$ точки $x_{0}$. Но тогда для всех $x \in U$ найдется такое $t(x) \in(0,1)$, что $v(t(x), x)=y$, следовательно, $U \subset D_{y}$. Если же $t_{0} \in\{0,1\}$, то точка $\left(t_{0}, x_{0}\right) \in \partial \Omega$ и по определению регулярного значения выполнены свойства: $x_{0} \in(0,1)$ (иначе $\left(t_{0}, x_{0}\right)$ является вершиной квадрата $\bar{\Omega}$ ), $v_{t}\left(t_{0}, x_{0}\right) \neq 0, v_{x}\left(t_{0}, x_{0}\right) \neq 0$. Пусть для определенности $t_{0}=0, v_{x}\left(t_{0}, x_{0}\right)<0$ (остальные случаи разбираются аналогично). Ясно, что $v\left(1, x_{0}\right)>v\left(0, x_{0}\right)=y$. Отсюда и из условия $v_{x}\left(0, x_{0}\right)<0$ следует, что $v(0, x)<y<v(1, x)$ для всех $x \in\left(x_{0}, x_{0}+h\right]$ при достаточно малом $h>0$. Но тогда $\left[x_{0}, x_{0}+h\right] \in D_{y}$. Итак, в любом из рассмотренных случаев точка $x_{0}$ входит в $D_{y}$ вместе с некоторым отрезком положительной длины. В частности, компоненты связности множества $D_{y}$ не могут состоять лишь из одной точки, т.е. являются невырожденными отрезками (ввиду их связности и компактности), что и утверждалось.

Если $[a, b]$ - один из таких отрезков, то кривая $C_{y}=\{(t(x), x): x \in[a, b]\}$ является компонентой связности прообраза $v^{-1}(y)$, при этом отображение $\gamma(x)=$ $(t(x), x)$ осуществляет гомеоморфизм отрезка $[a, b]$ на $C_{y}$ и концевые точки $\gamma(a), \gamma(b) \in \partial \Omega$ (последнее легко следует из рассуждений выше). Заметим также, что по регулярности $y$ для п.в. $x \in[a, b](t(x), x) \notin S$ и функция $t(x)$ имеет производную

$$
t^{\prime}(x)=-\frac{v_{x}(t, x)}{v_{t}(t, x)}=\frac{u(t, x)}{\varphi(u(t, x))}, \quad t=t(x) .
$$

Покажем, что функция $t(x)$ строго монотонна. Предположим противное. Тогда найдутся значения $x_{1}, x_{2} \in(a, b), x_{1}<x_{2}$, такие, что $t\left(x_{1}\right)=t\left(x_{2}\right)=\tau$. Далее, $t(x)$ не равно тождественно $\tau$ на $\left[x_{1}, x_{2}\right]$. В противном случае $t^{\prime}(x)=0$ на $\left(x_{1}, x_{2}\right)$, а это означает, что $v_{t}=v_{x}=0$ на соответствующем отрезке $\{\tau\} \times\left[x_{1}, x_{2}\right] \in C_{y}$. Поскольку $\mathscr{H}^{1}$-мера этого отрезка положительна, это противоречит регулярности $y$.

Итак, в точке экстремума $\bar{x} \in\left(x_{1}, x_{2}\right)$ функции $t(x)$ имеем $t(\bar{x}) \neq \tau$, пусть для определенности $t(\bar{x})>\tau$.

Ясно, что участок кривой $C_{y}$, соответствующий $x \in\left[x_{1}, x_{2}\right]$, лежит в $\Omega$ (иначе $(t(\bar{x}), \bar{x}) \in \partial \Omega$ и, как нетрудно проверить, в этом случае $v_{t}=v_{x}=0$ в точке $(t(\bar{x}), \bar{x})$, а это противоречит регулярности $y)$. Поэтому найдется достаточно малое $h>0$ такое, что $h<t(\bar{x})-\tau$ и $(t(x)+h, x) \in \Omega$ при $x \in\left[x_{1}, x_{2}\right]$.

Тогда $\alpha=\min _{x \in\left[x_{1}, x_{2}\right]} v(t(x)+h, x)-y>0$ и для любого $z \in(y, y+\alpha) \cap Y$ найдется кривая уровня $C_{z}=\left\{\left(t_{z}(x), x\right): x \in\left[x_{1}, x_{2}\right]\right\} \subset v^{-1}(z)$, причем $t(x)<$ $t_{z}(x)<t(x)+h$. 
Зададим функцию

$$
g: Y \cap(y, y+\alpha) \ni z \mapsto \max _{x \in\left[x_{1}, x_{2}\right]} t_{z}(x)
$$

Из условия $h<t(\bar{x})-\tau$ следует, что для произвольного $z \in Y \cap(y, y+\alpha)$ справедливы неравенства $\max _{x \in\left[x_{1}, x_{2}\right]} t_{z}(x)>t(\bar{x})>\tau+h>\max \left(t_{z}\left(x_{1}\right), t_{z}\left(x_{2}\right)\right)$ и максимум в определении функции $g$ достигается в некоторой внутренней точке $x_{0} \in\left(x_{1}, x_{2}\right)$, так что $g(z)=t_{z}\left(x_{0}\right)$. Ясно, что $v_{x}\left(t_{0}, x_{0}\right)=0$, где $t_{0}=t_{z}\left(x_{0}\right)$ (иначе $t_{z}(x)$ строго монотонна в окрестности $x_{0}$ ). Тогда также и $v_{t}\left(t_{0}, x_{0}\right)=0$. Отсюда легко вычисляется, что правосторонняя производная $g_{+}^{\prime}(z)=+\infty$, т.е.

$$
\frac{g\left(z_{1}\right)-g(z)}{z_{1}-z} \rightarrow+\infty \quad \text { при } z_{1} \in Y \cap(y, y+\alpha), \quad z_{1}>z_{0} .
$$

Действительно, если $\Delta t>0$ таково, что $z_{1}=v\left(t_{0}+\Delta t, x_{0}\right) \in Y \cap(y, y+\alpha)$, то $g\left(z_{1}\right) \geqslant t_{0}+\Delta t$, откуда $g\left(z_{1}\right)-g(z) \geqslant \Delta t$. Учитывая также, что $z_{1}-z=$ $v\left(t_{0}+\Delta t, x_{0}\right)-v\left(t_{0}, x_{0}\right)>0$, получаем соотношение

$$
\frac{g\left(z_{1}\right)-g(z)}{z_{1}-z} \geqslant\left(\frac{v\left(t_{0}+\Delta t, x_{0}\right)-v\left(t_{0}, x_{0}\right)}{\Delta t}\right)^{-1} \underset{\Delta t \rightarrow 0}{\longrightarrow}+\infty
$$

ввиду условия $v_{t}\left(t_{0}, x_{0}\right)=0$. Поэтому $g_{+}^{\prime}(z)=+\infty \forall z \in Y \cap(y, y+\alpha)$, что и утверждалось. Однако функция $g$ строго возрастает в силу своего определения и, значит, почти всюду дифференцируема (мы можем продолжить $g(z)$ по монотонности на весь интервал $(y, y+\alpha)$ и воспользоваться классической теоремой Лебега). Полученное противоречие завершает доказательство свойства строгой монотонности функции $t(x)$.

Обозначим

$$
\begin{aligned}
\Omega_{+}= & \left\{(t, x) \in \Omega: v_{x}(t, x)>0\right\}, \\
\Omega_{+0}= & \operatorname{Int}\left\{(t, x) \in \Omega: v_{x}(t, x) \geqslant 0\right\}, \\
\Omega_{-}= & \left\{(t, x) \in \Omega: v_{x}(t, x)<0\right\}, \\
& S_{0}=\mathrm{Cl} \Omega_{+} \cap \mathrm{Cl} \Omega_{-}
\end{aligned}
$$

(замыкания берутся относительно $\Omega$ ). Очевидно, $\Omega_{+} \subset \Omega_{+0}, S_{0} \subset S$. Докажем, что справедливо свойство

$$
v^{-1}(y) \cap S_{0}=\varnothing \quad \forall y \in Y .
$$

В противном случае найдется кривая $C_{y}=\{(t(x), x): x \in[a, b]\}$ такая, что $C_{y} \cap S_{0} \neq \varnothing$. Как установлено выше, функция $t(x)$ строго монотонна. Предположим для определенности, что $t(x)$ строго убывает. В частности, $\tau_{2}=$ $t(b)<\tau_{1}=t(a)$. Так как $C_{y} \cap S_{0} \neq \varnothing$, то найдется точка $\left(t_{1}, x_{1}\right) \in\left(\tau_{2}, \tau_{1}\right) \times(a, b)$ такая, что $v_{x}\left(t_{1}, x_{1}\right)<0, v\left(t_{1}, x_{1}\right)=z \in Y$ и $z \neq y$. Пусть $C_{z}=\left\{\left(t_{z}(x), x\right): x \in\right.$ $\left.\left[a_{1}, b_{1}\right]\right\}$ - компонента связности $v^{-1}(z)$, содержащая точку $\left(t_{1}, x_{1}\right)$. Тогда, как следует из (31) и строгой монотонности функции $t_{z}(x), t_{z}(x)$ строго возрастает. Заметим также, что $\left(t_{z}\left(a_{1}\right), a_{1}\right),\left(t_{z}\left(b_{1}\right), b_{1}\right) \in \partial \Omega$. Из указанных свойств кривых $C_{y}, C_{z}$ легко вывести, что эти кривые должны пересекаться, а это невозможно. Полученное противоречие доказывает свойство (Р). 
Перейдем к доказательству основного соотношения (27), которое при $k=0$ означает, что $\forall f \in C_{0}^{\infty}(\Omega)$

$$
\int_{\Omega}\left[u^{+} f_{t}+\varphi\left(u^{+}\right) f_{x}\right] d t d x=0
$$

Пусть $f(t, x) \in C_{0}^{\infty}(\Omega), K=\operatorname{supp} f$. Рассмотрим компакт $K_{0}=K \cap S_{0}$ и положим $B=v\left(K_{0}\right), A=v^{-1}(B) \cap \Omega_{+}$. Ввиду свойства $(\mathrm{P}) \quad Y \cap v(A)=\varnothing$ и $v(A)$ имеет нулевую меру Лебега. Тогда по формуле коплощади

$$
\int_{A}|\nabla v(t, x)| d t d x=\int_{\mathbb{R}} \mathscr{H}^{1}\left(v^{-1}(y) \cap A\right) d y=\int_{v(A)} \mathscr{H}^{1}\left(v^{-1}(y) \cap A\right) d y=0 .
$$

Так как $|\nabla v(t, x)|>0$ на $\Omega_{+}$, то meas $(A)=0$, где meas $(A)$ обозначает меру Лебега измеримого множества $A$ (мы используем общее обозначение $\operatorname{meas}(A)$ для всех размерностей).

Пусть при $r>0$

$$
B_{r}=\left\{y \in \mathbb{R}: \operatorname{dist}(y, B)=\inf _{z \in B}|y-z|<r\right\}
$$

- $r$-окрестность множества $B, A_{r}=v^{-1}\left(B_{r}\right) \cap \Omega_{+}$. Множество $B$ замкнуто (как непрерывный образ компакта) и, значит, $B=\bigcap_{r>0} B_{r}$, откуда и $A=\bigcap_{r>0} A_{r}$. Фиксируем $\varepsilon>0$. Поскольку meas $(A)=0$, то $\exists r>0$ : $\operatorname{meas}\left(A_{r}\right)<\varepsilon$. Следует подчеркнуть, что при $S_{0}=\varnothing$ множества $B_{r}, A_{r}$ пусты при всех $r>0$.

Выберем далее функцию $\rho(y) \in C_{0}^{\infty}(\mathbb{R})$ такую, что $0 \leqslant \rho(y) \leqslant 1, \operatorname{supp} \rho \subset B_{r}$, $\rho=1$ в окрестности $B_{r / 2}$ множества $B$, и положим

$$
g=g(t, x)=f(t, x) \rho(v(t, x)), \quad h=h(t, x)=f(t, x)[1-\rho(v(t, x))] .
$$

Ясно, что $g, h \in C_{0}^{1}(\Omega), f=g+h$ (и $g=0$ при $\left.S_{0}=\varnothing\right)$. Поэтому интеграл в левой части (32) перепишется в виде

$$
I=\int_{\Omega}\left[u^{+} f_{t}+\varphi\left(u^{+}\right) f_{x}\right] d t d x=I_{1}+I_{2}
$$

где $u^{+}=\max (u, 0)$ и

$$
I_{1}=\int_{\Omega_{+}}\left[u g_{t}+\varphi(u) g_{x}\right] d t d x, \quad I_{2}=\int_{\Omega_{+0}}\left[u h_{t}+\varphi(u) h_{x}\right] d t d x .
$$

Заметим, что

$$
K_{1}=\left(K \cap \mathrm{Cl} \Omega_{+}\right) \backslash v^{-1}\left(B_{r / 2}\right) \subset \mathrm{Cl} \Omega_{+} \backslash S_{0}=\mathrm{Cl} \Omega_{+} \backslash \mathrm{Cl} \Omega_{-} \subset \Omega \backslash \mathrm{Cl} \Omega_{-}=\Omega_{+0},
$$

откуда следует, что существует функция $\eta(t, x) \in C^{1}(\Omega)$ такая, что $\eta(t, x)=1$ на компакте $K_{1}$ и $\operatorname{supp} \eta \subset \Omega_{+0}$. Положим $h_{1}(t, x)=h(t, x) \eta(t, x), h_{2}(t, x)=$ $h(t, x)(1-\eta(t, x))$. Тогда $h=h_{1}+h_{2}$ и $I_{2}=I_{3}+I_{4}$, где

$$
I_{3}=\int_{\Omega_{+0}}\left[u\left(h_{1}\right)_{t}+\varphi(u)\left(h_{1}\right)_{x}\right] d t d x, \quad I_{4}=\int_{\Omega_{+}}\left[u\left(h_{2}\right)_{t}+\varphi(u)\left(h_{2}\right)_{x}\right] d t d x .
$$

Так как $h_{1}(t, x) \in C_{0}^{1}\left(\Omega_{+0}\right)$, а $u(t, x)$ - о.р. уравнения $(24)$ в $\Omega_{+0}$, то первый интеграл $I_{3}=0$. Далее, как легко видеть, supp $h \cap \Omega_{+} \subset K_{1}$, откуда следует, 
что $h_{2}=0$ на $\Omega_{+}$. Поэтому также и $I_{4}=0$. Итак, $I_{2}=0$. Следует отметить, что при $S_{0}=\varnothing$ интеграл $I=I_{2}$ и требуемое соотношение $I=0$ доказано. В общем случае заметим, что при $v=v(t, x), u=u(t, x)$

$$
\begin{aligned}
g_{t} & =f_{t} \rho(v)+f \rho^{\prime}(v) v_{t}=f_{t} \rho(v)-f \rho^{\prime}(v) \varphi(u), \\
g_{x} & =f_{x} \rho(v)+f \rho^{\prime}(v) v_{x}=f_{x} \rho(v)+f \rho^{\prime}(v) u .
\end{aligned}
$$

Подставляя эти выражения в интеграл $I_{1}$ и учитывая, что $\rho(v)=0$ вне $A_{r}$, получаем равенство

$$
I_{1}=\int_{\Omega_{+}}\left[u f_{t}+\varphi(u) f_{x}\right] \rho(v) d t d x=\int_{A_{r}}\left[u f_{t}+\varphi(u) f_{x}\right] \rho(v) d t d x .
$$

Следовательно,

$$
\left|I_{1}\right| \leqslant \int_{A_{r}}\left[|u|\left|f_{t}\right|+|\varphi(u)|\left|f_{x}\right|\right] d t d x \leqslant c \operatorname{meas}\left(A_{r}\right)<c \varepsilon,
$$

где $c$ - константа, не зависящая от $\varepsilon$. Тогда $|I|=\left|I_{1}\right|<c \varepsilon$ и с учетом произвольности $\varepsilon>0 \quad I=0$, т.е. выполнено условие (32). Теорема доказана.

Оказывается, в случае $\Omega=\Pi_{T}, 0<T \leqslant \infty$, для существования и.р. необходимо, чтобы функция потока $\varphi(u)$ была непрерывна по Липшицу в области значений этого решения. Точнее, справедлива следующая

ТеОрема 4. Пусть $u(t, x) \in L_{\mathrm{loc}}^{\infty}\left(\Pi_{T}\right)$ является и.р. уравнения (24) в области $\Omega=\Pi_{T} ;-\infty \leqslant m=\operatorname{essinf} u(t, x), M=\operatorname{ess} \sup u(t, x) \leqslant+\infty$ в $\Omega$. Тогда функиия $\varphi$ удовлетворяет условию Липшица на любом отрезке $[a, b] \subset(m, M)$.

ДокАЗАТЕЛЬство. Доказательство теоремы проведем методом от противного. Предположим, что $\varphi(u)$ не удовлетворяет условию Липшица на некотором отрезке $[a, b] \subset(m, M)$. Тогда найдутся последовательности $a_{n}, b_{n} \in[a, b]$, $a_{n}<b_{n}, n \in \mathbb{N}$, такие, что

$$
\left|\varphi\left(b_{n}\right)-\varphi\left(a_{n}\right)\right|=\max _{u \in\left[a_{n}, b_{n}\right]}\left|\varphi(u)-\varphi\left(a_{n}\right)\right|>n\left|b_{n}-a_{n}\right| .
$$

Очевидно, $b_{n}-a_{n} \rightarrow 0$ при $n \rightarrow \infty$ и, после возможного перехода к подпоследовательности, $a_{n} \rightarrow \alpha, b_{n} \rightarrow \alpha$, где $\alpha \in[a, b]$. Обозначим $C_{n}=\varphi\left(b_{n}\right)-\varphi\left(a_{n}\right)$ и выберем "энтропии"

$$
p_{n}(u)=\frac{\left|u-a_{n}\right|-\left|u-b_{n}\right|+b_{n}-a_{n}}{2 C_{n}}= \begin{cases}0, & u<a_{n}, \\ \frac{u-a_{n}}{C_{n}}, & u \in\left[a_{n}, b_{n}\right], \\ \frac{b_{n}-a_{n}}{C_{n}}, & u>b_{n},\end{cases}
$$

с соответствующими потоками

$$
\begin{aligned}
q_{n}(u)= & \frac{1}{2 C_{n}}\left[\left(\varphi(u)-\varphi\left(a_{n}\right)\right) \operatorname{sign}\left(u-a_{n}\right)\right. \\
& \left.\quad-\left(\varphi(u)-\varphi\left(b_{n}\right)\right) \operatorname{sign}\left(u-b_{n}\right)+\varphi\left(b_{n}\right)-\varphi\left(a_{n}\right)\right] \\
= & \begin{cases}0, & u<a_{n}, \\
\frac{\varphi(u)-\varphi\left(a_{n}\right)}{C_{n}}, & u \in\left[a_{n}, b_{n}\right], \\
1, & u>b_{n} .\end{cases}
\end{aligned}
$$


Тогда из (6) следует, что $\forall n \in \mathbb{N}$

$$
p_{n}(u)_{t}+q_{n}(u)_{x}=0 \quad \text { в } \mathscr{D}^{\prime}(\Omega) .
$$

Ввиду (33) имеем

$$
0 \leqslant\left|p_{n}(u)\right| \leqslant \frac{b_{n}-a_{n}}{\left|C_{n}\right|}<\frac{1}{n}, \quad\left|q_{n}(u)\right| \leqslant 1 .
$$

После дополнительного выделения подпоследовательности мы можем считать, что $q_{n}(\alpha) \rightarrow \beta \in[-1,1]$ при $n \rightarrow \infty$. Тогда $q_{n}(u) \rightarrow \theta(u-\alpha) \forall u \in \mathbb{R}$, где

$$
\theta(v)= \begin{cases}1, & v>0 \\ \beta, & v=0 \\ 0, & v<0\end{cases}
$$

С учетом приведенных выше соотношений из (34) в пределе при $n \rightarrow \infty$ следует, что

$$
\theta(u-\alpha)_{x}=0 \quad \text { в } \quad \mathscr{D}^{\prime}(\Omega) .
$$

Из этого соотношения следует, что для п.в. $t \in(0, T) \theta(u(t, \cdot)-\alpha)_{x}=0$ в $\mathscr{D}^{\prime}(\mathbb{R})$. В частности, это равенство выполнено при $t \in E$, где $E \subset(0, T)$ - множество полной меры, для которого выполнены утверждения предложения 2. По предложению 2 имеем $m=\operatorname{essinf} u(t, \cdot), M=\operatorname{ess} \sup u(t, \cdot)$ при $t \in E$ и условие $\alpha \in(m, M)$ противоречит равенству $\theta(u(t, \cdot)-\alpha)_{x}=0$ в $\mathscr{D}^{\prime}(\mathbb{R})$.

Полученное противоречие завершает доказательство теоремы.

В предыдущей теореме существенно, что область $\Omega=\Pi_{T}$. Как будет показано ниже в примере, для произвольной области $\Omega$ утверждение теоремы 4 неверно. В общем случае справедлива следующая

ТЕорема 5. Пусть и $(t, x)$ является и.р. уравнения $(24)$ в области $\Omega, m=$ $\operatorname{ess} \inf u(t, x), M=\operatorname{ess} \sup u(t, x)$ в $\Omega$. Тогда найдется замкнутое относительно интервала $(m, M)$ множество $F$ нулевой меры Лебега такое, что функция $\varphi$ удовлетворяет условию Липшица локально на дополнении $(m, M) \backslash F$.

ДокАзАтельство. В случае, когда и.р. $u(t, x)$ постоянно, $m=M$ и утверждение теоремы тривиально. Поэтому предположим, что $m<M$, и рассмотрим множество $U$, состоящее из значений $u \in(m, M)$ таких, что $\varphi(u)$ удовлетворяет условию Липшица в некоторой окрестности точки $u$. По построению $U$ открыто и $\varphi(u)$ удовлетворяет условию Липшица локально на $U$. Положим $F=(m, M) \backslash U$. Тогда $F$ замкнуто в $(m, M)$ и для завершения доказательства остается показать, что $F$ имеет нулевую меру. Пусть $E=\{v \in(m, M)$ : meas $\left.u^{-1}(v)=0\right\}$. Поскольку график $\Gamma$ функции $u(t, x)$ имеет нулевую меру, то для п.в. $v \in(m, M)$ сечения $\Gamma^{v}=\{(t, x) \in \Omega:(t, x, v) \in \Gamma\}=u^{-1}(v)$ имеют нулевую меру на $\Omega$ и, следовательно, $E$ является множеством полной меры на $(m, M)$. Достаточно доказать, что множество $F_{1}=F \cap E$ имеет нулевую меру. Последнее, в свою очередь, достаточно проверить локально, т.е. мы должны установить, что $\forall v \in F_{1} \exists V$ - окрестность $v$ такая, что $\operatorname{meas}\left(F_{1} \cap V\right)=0$.

Итак, пусть $v_{0} \in F_{1}$. Рассмотрим множества

$$
\Omega^{+}=\operatorname{ess} \operatorname{Cl}\left\{(t, x) \in \Omega: u(t, x)<v_{0}\right\}, \quad \Omega^{-}=\operatorname{ess} \operatorname{Cl}\left\{(t, x) \in \Omega: u(t, x)>v_{0}\right\},
$$


где существенное замыкание ess $\mathrm{Cl}(A)$ множества $A$ состоит из точек, любая окрестность которых пересекает $A$ по множеству положительной меры. Очевидно, ess $\mathrm{Cl}(A)$ является замкнутым множеством. Действительно, его дополнение - это открытое множество, каждая из точек которого имеет окрестность, пересекающуюся с $A$ по множеству нулевой меры. Учитывая, что $v_{0} \in E$, получаем, что объединение $\Omega^{+} \cup \Omega^{-}=\Omega$, в противном случае $u(t, x)=v_{0}$ п.в. на открытом множестве $\Omega \backslash\left(\Omega^{+} \cup \Omega^{-}\right)$и meas $u^{-1}\left(v_{0}\right)>0$. Ввиду связности $\Omega$ найдется $\left(t_{0}, x_{0}\right) \in \Omega^{+} \cap \Omega^{-}$. Тогда в любой окрестности $V$ точки $\left(t_{0}, x_{0}\right)$ множества

$$
\left\{(t, x) \in \Omega: u(t, x)<v_{0}\right\}, \quad\left\{(t, x) \in \Omega: u(t, x)>v_{0}\right\}
$$

имеют положительную меру. Выберем квадратную окрестность $V=V_{t} \times V_{x}$, где $V_{t}=\left(t_{0}-\varepsilon, t_{0}+\varepsilon\right), V_{x}=\left(x_{0}-\varepsilon, x_{0}+\varepsilon\right)$, а $\varepsilon>0$ достаточно мало, так что $V \subset \Omega$ и $u(t, x) \in L^{\infty}(V)$. Положим $m_{1}=\operatorname{essinf}_{V} u(t, x), M_{1}=\operatorname{ess}_{\sup _{V}} u(t, x)$. Тогда $m \leqslant m_{1}<v_{0}<M_{1} \leqslant M$ и $I=\left(m_{1}, M_{1}\right)$ является окрестностью точки $v_{0}$. Покажем, что meas $\left(F_{1} \cap I\right)=0$. Пусть $\rho(x) \in C_{0}^{\infty}\left(V_{x}\right), \rho(x) \geqslant 0, \rho\left(x_{0}\right)>0$. Положим

$$
F(t)=\frac{1}{A} \int_{x_{0}-\varepsilon}^{x_{0}+\varepsilon} u(t, x) \rho(x) d x, \quad G(t)=\frac{1}{A} \int_{x_{0}-\varepsilon}^{x_{0}+\varepsilon} \varphi(u(t, x)) \rho^{\prime}(x) d x,
$$

где

$$
A=\int_{x_{0}-\varepsilon}^{x_{0}+\varepsilon} \rho(x) d x>0 .
$$

Функции $F(t), G(t)$ определены почти всюду на интервале $V_{t}$. В частности, эти функции определены на множестве полной меры $J \subset V_{t}$ значений $t$ таких, что для п.в. $x \in V_{x} \quad(t, x)$ - точка Лебега $u(t, x)$. Ясно, что значения $t \in J$ являются также точками Лебега функций $F(t), G(t)$. Из равенства $u_{t}+\varphi(u)_{x}=0$ в $\mathscr{D}^{\prime}(V)$ следует, что $F^{\prime}(t)=G(t) \in L^{\infty}$ в $\mathscr{D}^{\prime}\left(V_{t}\right)$. В частности, $F(t)$ удовлетворяет условию Липшица на $V_{t}$ (после возможной коррекции на множестве нулевой меры). Кроме того, ясно, что при $t \in J$ существует классическая производная $F^{\prime}(t)=G(t)$. Поскольку $F(t)$ удовлетворяет условию Липшица, образ $\mathscr{E}=F\left(V_{t} \backslash J\right)$ имеет нулевую меру. Это непосредственно следует из формулы площади (см. [9; теорема 3.2.3])

$$
0=\int_{V_{t} \backslash J}\left|F^{\prime}(t)\right| d t=\int \mathscr{H}_{0}\left(F^{-1}(v) \cap\left(V_{t} \backslash J\right)\right) d v \geqslant \operatorname{meas}(\mathscr{E}) .
$$

Здесь $\mathscr{H}_{0}$ - нульмерная мера Хаусдорфа (т.е. считающая мера).

Пусть $I_{1}=I \backslash \mathscr{E}$. Если $c \in F_{1} \cap I_{1}$, то функция $\varphi(u)$ не удовлетворяет условию Липшица ни в какой окрестности точки $c$ и, значит, найдутся последовательности $a_{n}, b_{n} \in[a, b], a_{n}<b_{n}$, такие, что $a_{n}, b_{n} \rightarrow c$ при $n \rightarrow \infty$ и

$$
\left|\varphi\left(b_{n}\right)-\varphi\left(a_{n}\right)\right|=\max _{u \in\left[a_{n}, b_{n}\right]}\left|\varphi(u)-\varphi\left(a_{n}\right)\right|>n\left|b_{n}-a_{n}\right|, \quad n \in \mathbb{N} .
$$

Определим последовательность энтропий $p_{n}(u)$ и соответствующую последовательность потоков $q_{n}(u)$ так же, как в доказательстве теоремы 4 , и перейдем в энтропийном соотношении вида (34) к пределу при $n \rightarrow \infty$. Получим, что

$$
\theta(v-c)_{x}=0 \quad \text { в } \quad \mathscr{D}^{\prime}(V),
$$


где функция $\theta(v)$ определена так же, как в доказательстве теоремы 4. Из этого соотношения следует, что для $t \in J$ выполнено одно из следующих соотношений:

1) $u(t, x) \geqslant c$ п.в. на $V_{x}$;

2) $u(t, x) \leqslant c$ п.в. на $V_{x}$.

Заметим, что эти соотношения эквивалентны неравенствам $F(t) \geqslant c, F(t) \leqslant c$ соответственно. Оба замкнутых множества, на которых выполнены эти неравенства, непусты, в противном случае $c \notin I$. Следовательно, найдется значение $\bar{t} \in V_{t}$, для которого $F(\bar{t})=c$. Из условия $c \notin \mathscr{E}$ следует, что $\bar{t} \in J$. Тогда $u(\bar{t}, x)=c$ п.в. на $V_{x}$ и

$$
F^{\prime}(\bar{t})=G(\bar{t})=\mathrm{const} \int \rho^{\prime}(x) d x=0 .
$$

Поэтому любое значение $c \in F_{1} \cap I_{1}$ является критическим для $F(t)$, т.е. при $J_{0}=J \cap G^{-1}(0)$ пересечение $F^{-1}(c) \cap J_{0} \neq \varnothing$. Кроме того, $F^{\prime}(t)=G(t)=0$ при $t \in J_{0}$ и по формуле площади

$$
0=\int_{J_{0}}\left|F^{\prime}(t)\right| d t=\int \mathscr{H}_{0}\left(F^{-1}(c) \cap J_{0}\right) d c \geqslant \operatorname{meas}\left(F_{1} \cap I_{1}\right) .
$$

Итак, meas $\left(F_{1} \cap I\right)=\operatorname{meas}\left(F_{1} \cap I_{1}\right)=0$, что и требовалось доказать.

Теорема 5 точна в том смысле, что множество $F \subset(m, M)$ "плохих" точек может быть произвольным замкнутым множеством нулевой меры. Подтвердим это следующим примером, в котором соответствующее и.р. определено и непрерывно на замыкании прямоугольной области $\Omega=(0, T) \times(0,1)$.

Пример. Пусть $m<M$ и $F \subset(m, M)$ - замкнутое множество нулевой меры. Построим сначала функцию потока $\varphi(u) \in C(\mathbb{R})$, для которой $F$ является множеством "плохих" точек на $(m, M)$, т.е. $F$ - минимальное замкнутое в $(m, M)$ множество такое, что на его дополнении $U=(m, M) \backslash F$ функция $\varphi(u)$ удовлетворяет локально условию Липшица. Множество $U$ является объединением не более чем счетного семейства попарно непересекающихся интервалов. Рассмотрим более сложный случай, когда это семейство счетно: $U=\bigcup_{i \in \mathbb{N}}\left(a_{i}, b_{i}\right)$. Случай конечного набора интервалов $\left(a_{i}, b_{i}\right)$ соответствует конечному множеству $F$ и разбирается аналогично с очевидными упрощениями. Положим $h_{i}=b_{i}-a_{i}, c_{i}=\left(a_{i}+b_{i}\right) / 2$. Тогда $\sum_{i=1}^{\infty} h_{i}=M-m<+\infty$, откуда легко следует существование убывающей последовательности $\alpha_{i}>0$, $i \in \mathbb{N}$, такой, что $\alpha_{i} \rightarrow 0$ при $i \rightarrow \infty$ и

$$
T=8 \sum_{i=1}^{\infty} \frac{h_{i}}{\alpha_{i}}<+\infty
$$

Рассмотрим при $i \in \mathbb{N}$ неотрицательные непрерывные функции

$$
\varphi_{i}(u)= \begin{cases}\frac{1}{\sqrt{2 h_{i}}} \min \left(\sqrt{u-a_{i}}, \sqrt{b_{i}-u}\right), & u \in\left(a_{i}, b_{i}\right), \\ 0, & u \notin\left(a_{i}, b_{i}\right) .\end{cases}
$$

Ясно, что $\varphi_{i}(u)$ удовлетворяет условию Липшица в окрестности любой точки, за исключением точек $a_{i}, b_{i}$. Кроме того, очевидно, что $\max \varphi_{i}(u)=\varphi_{i}\left(c_{i}\right)=\frac{1}{2}$. 
Положим $\varphi(u)=\sum_{i=1}^{\infty} \alpha_{i} \varphi_{i}(u)$, так что $\varphi(u)=\alpha_{i} \varphi_{i}(u)$ на интервалах $\left(a_{i}, b_{i}\right)$ и $\varphi(u)=0$ на $\mathbb{R} \backslash U$. Очевидно,

$$
0 \leqslant \sum_{i=m}^{\infty} \alpha_{i} \varphi_{i}(u) \leqslant \frac{\alpha_{m}}{2} \underset{m \rightarrow \infty}{\longrightarrow} 0
$$

при $m \rightarrow \infty$. Поэтому ряд, определяющий функцию $\varphi(u)$, сходится равномерно на $\mathbb{R}$, и, значит, эта функция непрерывна. Кроме того, ясно, что $\varphi(u)$ удовлетворяет условию Липшица локально на $U$, а все точки множества $F$ являются "плохими". Последнее следует из того факта, что любая окрестность $V$ точки $u \in F$ пересекается с множеством $\bigcup_{i \in \mathbb{N}}\left\{a_{i}, b_{i}\right\}$ (в противном случае $V \subset F$, а это противоречит требованию meas $(F)=0)$ и, следовательно, $\varphi(u)$ не может удовлетворять условию Липшица на $V$. Итак, множество "плохих" точек функции $\varphi(u)$ на интервале $(m, M)$ совпадает с $F$.

Перейдем к построению решения $u(t, x)$. Рассмотрим ступенчатую функцию $\rho(u)$ на $(m, M)$, равную $8 / \alpha_{i}$ на интервалах $\left(a_{i}, b_{i}\right)$ и нулю на множестве $F$. Положим

$$
g(u)=\int_{m}^{u} \rho(v) d v .
$$

Ясно, что $g(u)$ абсолютно непрерывна (так как по условию $(35) \int_{m}^{M} \rho(v) d v=$ $T<+\infty)$ и строго возрастает на отрезке $[m, M], g(m)=0, g(M)=T$. Обозначим $A_{i}=g\left(a_{i}\right), B_{i}=g\left(b_{i}\right)$. Ясно, что $B_{i}-A_{i}=H_{i}=8 h_{i} / \alpha_{i}$. Непосредственно проверяется (с учетом равенств $\alpha_{i} H_{i}=8 h_{i}$ ), что функции

$$
v_{i}(t, x)=a_{i}+\frac{\alpha_{i}\left(t-A_{i}\right)^{2}}{H_{i}(x+1)^{2}}, \quad w_{i}(t, x)=b_{i}-\frac{\alpha_{i}\left(B_{i}-t\right)^{2}}{H_{i}(x+1)^{2}}
$$

являются гладкими автомодельными решениями уравнений

$$
u_{t}+\frac{\alpha_{i}}{\sqrt{2 h_{i}}}\left(\sqrt{u-a_{i}}\right)_{x}=0, \quad u_{t}+\frac{\alpha_{i}}{\sqrt{2 h_{i}}}\left(\sqrt{b_{i}-u}\right)_{x}=0
$$

соответственно в замкнутых областях

$$
\begin{aligned}
& P_{i}^{-}=\left\{(t, x): x \in[0,1], A_{i} \leqslant t \leqslant A_{i}+\frac{(x+1) H_{i}}{4}\right\}, \\
& P_{i}^{+}=\left\{(t, x): x \in[0,1], B_{i}-\frac{(x+1) H_{i}}{4} \leqslant t \leqslant B_{i}\right\},
\end{aligned}
$$

причем $v_{i}(t, x) \in\left[a_{i}, c_{i}\right]$ на $P_{i}^{-}, v_{i}\left(A_{i}, x\right)=a_{i}, v_{i}(t, x)=c_{i}$ при $t=A_{i}+(x+1) \times$ $H_{i} / 4$, а $w_{i}(t, x) \in\left[c_{i}, b_{i}\right]$ на $P_{i}^{+}, w_{i}\left(B_{i}, x\right)=b_{i}, w_{i}(t, x)=c_{i}$ при $t=B_{i}-(x+1) \times$ $H_{i} / 4$. Учитывая, что по построению

$$
\begin{array}{lll}
\varphi(u)=\frac{\alpha_{i}}{\sqrt{2 h_{i}}} \sqrt{u-a_{i}} & \text { при } \quad u \in\left[a_{i}, c_{i}\right], \\
\varphi(u)=\frac{\alpha_{i}}{\sqrt{2 h_{i}}} \sqrt{b_{i}-u} & \text { при } \quad u \in\left[c_{i}, b_{i}\right],
\end{array}
$$

заключаем, что $v_{i}(t, x), w_{i}(t, x)$ - гладкие решения уравнения $(24)$. 
Рассмотрим функцию $u_{i}(t, x) \in\left[a_{i}, b_{i}\right]$, заданную на замыкании прямоугольной области $P_{i}=\left(A_{i}, B_{i}\right) \times(0,1)$ равенством

$$
u_{i}(t, x)= \begin{cases}v_{i}(t, x), & (t, x) \in P_{i}^{-} \\ w_{i}(t, x), & (t, x) \in P_{i}^{+}, \\ c_{i}, & (t, x) \in P_{i} \backslash\left(P_{i}^{-} \cup P_{i}^{+}\right) .\end{cases}
$$

Заметим, что $P_{i} \backslash\left(P_{i}^{-} \cup P_{i}^{+}\right)$является треугольником:

$$
P_{i} \backslash\left(P_{i}^{-} \cup P_{i}^{+}\right)=\left\{(t, x): x \in[0,1], A_{i}+\frac{(x+1) H_{i}}{4}<t<B_{i}-\frac{(x+1) H_{i}}{4}\right\} .
$$

Равенство (36) определяет кусочно гладкое и непрерывное вплоть до границы области $P_{i}$ и.р. уравнения $(24)$. Искомое и.р. $u(t, x)$ в целом прямоугольнике $\Omega$ строится склеиванием решений $u_{i}(t, x)$. А именно положим

$$
u(t, x)= \begin{cases}u_{i}(t, x), & t \in\left(A_{i}, B_{i}\right) \\ g^{-1}(t), & t \in g(F)\end{cases}
$$

Заметим, что обратная функция $g^{-1}:[0, T] \mapsto[m, M]$ определена и непрерывна. Кроме того, множество $E=g(F)$ является компактным подмножеством нулевой меры. Действительно, ввиду абсолютной непрерывности $g(u)$

$$
\operatorname{meas}(E)=\int_{F} \rho(u) d u=0 .
$$

Ясно, что $(0, T)=E \cup \bigcup_{i \in \mathbb{N}}\left(A_{i}, B_{i}\right)$ и равенство (37) корректно определяет функцию $u(t, x)$ на $\Omega$. Покажем, что эта функция непрерывна. Пусть $h(t)=$ $g^{-1}(t)$. Тогда $u_{i}\left(A_{i}, x\right)=h\left(A_{i}\right)=a_{i}, u_{i}\left(B_{i}, x\right)=h\left(B_{i}\right)=b_{i}$, откуда следуют оценки $\left|u_{i}(t, x)-h(t)\right| \leqslant b_{i}-a_{i}=h_{i}, t \in\left[A_{i}, B_{i}\right]$. Тогда при

$$
p_{m}(t, x)= \begin{cases}u_{i}(t, x), & t \in\left(A_{i}, B_{i}\right), 1 \leqslant i \leqslant m \\ h(t), & t \in[0, T] \backslash \bigcup_{i=1}^{m}\left(A_{i}, B_{i}\right)\end{cases}
$$

имеем

$$
\left|u(t, x)-p_{m}(t, x)\right| \leqslant \max _{i>m} h_{i} \underset{m \rightarrow \infty}{\longrightarrow} 0,
$$

откуда следует равномерная сходимость последовательности непрерывных на $\mathrm{Cl} \Omega$ функций $p_{m}(t, x)$ к функции $u(t, x)$. Поэтому $u(t, x)$ непрерывна на $\Omega$ (и продолжается до непрерывной функции на $\mathrm{Cl} \Omega$ ). Осталось показать, что $u(t, x)$ является и.р. нашего уравнения. Для этого фиксируем $k \in \mathbb{R}$ и обозначим $\eta(u)=|u-k|, \psi(u)=\operatorname{sign}(u-k)(\varphi(u)-\varphi(k))$. Тогда для любой пробной функции $f=f(t, x) \in C_{0}^{\infty}(\Omega)$

$$
\begin{aligned}
\int_{\Omega}\left[\eta(u) f_{t}+\psi(u) f_{x}\right] d t d x & =\int_{\Omega \backslash E}\left[\eta(u) f_{t}+\psi(u) f_{x}\right] d t d x \\
& =\sum_{i=1}^{\infty} \int_{P_{i}}\left[\eta(u) f_{t}+\psi(u) f_{x}\right] d t d x
\end{aligned}
$$


Применяя формулу интегрирования по частям и учитывая, что $u=u(t, x)-$ кусочно гладкое и.р. на $P_{i}$, откуда $\eta(u)_{t}+\psi(u)_{x}=0$ в $\mathscr{D}^{\prime}\left(P_{i}\right)$, выводим равенство

$$
\begin{aligned}
\int_{P_{i}}\left[\eta(u) f_{t}+\psi(u) f_{x}\right] d t d x & =\int_{0}^{1} \eta\left(u\left(B_{i}, x\right)\right) f\left(B_{i}, x\right) d x-\int_{0}^{1} \eta\left(u\left(A_{i}, x\right)\right) f\left(A_{i}, x\right) d x \\
& =\eta\left(b_{i}\right) q\left(B_{i}\right)-\eta\left(a_{i}\right) q\left(A_{i}\right),
\end{aligned}
$$

где

$$
q(t)=\int_{0}^{1} f(t, x) d x \in C_{0}^{\infty}((0, T)) .
$$

Тогда из формулы (38) с учетом абсолютной непрерывности функции $g(u)$ и условия $\operatorname{meas}(F)=0$ получаем, что

$$
\begin{gathered}
\int_{\Omega}\left[\eta(u) f_{t}+\psi(u) f_{x}\right] d t d x=\sum_{i=1}^{\infty}\left(\eta\left(b_{i}\right) q\left(B_{i}\right)-\eta\left(a_{i}\right) q\left(A_{i}\right)\right) \\
=\sum_{i=1}^{\infty} \int_{a_{i}}^{b_{i}}(\eta(u) q(g(u)))^{\prime} d u=\int_{(m, M) \backslash F}(\eta(u) q(g(u)))^{\prime} d u \\
=\int_{m}^{M}(\eta(u) q(g(u)))^{\prime} d u=\eta(M) q(T)-\eta(m) q(0)=0 .
\end{gathered}
$$

Итак, $\forall f \in C_{0}^{\infty}(\Omega)$

$$
\int_{\Omega}\left[\eta(u) f_{t}+\psi(u) f_{x}\right] d t d x=0
$$

т.е. $\eta(u)_{t}+\psi(u)_{x}=0$ в $\mathscr{D}^{\prime}(\Omega)$. Последнее ввиду произвольности $k \in \mathbb{R}$ означает, что $u(t, x)$ - и.р. уравнения $(24)$, что и требовалось установить.

Перейдем к исследованию более тонких свойств функции потока $\varphi(u)$, вытекающих из условия существования нетривиального непрерывного и.р. Нам понадобится следующая

ЛЕмма 2. Пусть $f(y)$ - функиия на отрезке $[a, b]$,

$$
y(x) \in C([\alpha, \beta]), \quad y([\alpha, \beta])=[a, b]
$$

$u E \subset[a, b]$. Предположим, что композиция $g(x)=f(y(x))$ имеет ограни-

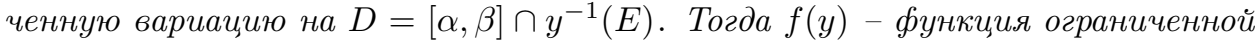
вариации на $E$, причем $\operatorname{Var}_{E} f(y) \leqslant \operatorname{Var}_{D} g(x)$.

ДокАЗАТЕЛЬство. Выберем значения $\alpha_{1}, \beta_{1} \in[\alpha, \beta]$ так, чтобы $y\left(\alpha_{1}\right)=a$, $y\left(\beta_{1}\right)=b$, и предположим для определенности, что $\alpha_{1}<\beta_{1}$ (случай $\alpha_{1}>\beta_{1}$ сводится к рассматриваемому случаю при замене функции $y(x)$ на $y(\alpha+\beta-x))$. Пусть $y_{i} \in E, i=1, \ldots, m,-$ набор точек, $y_{i}>y_{i-1}, i=2, \ldots, m$. Положим $x_{i}=\max \left\{x \in\left[\alpha_{1}, \beta_{1}\right]: y(x)=y_{i}\right\}$. Заметим, что по непрерывности $y(x)$ при $i>1 \quad y_{i} \in\left(y_{i-1}, b\right] \subset y\left(\left(x_{i-1}, \beta_{1}\right]\right)$ и, значит, $x_{i}>x_{i-1}, i=2, \ldots, m$. По построению $x_{i} \in D$. Тогда

$$
\sum_{i=2}^{m}\left|f\left(y_{i}\right)-f\left(y_{i-1}\right)\right|=\sum_{i=2}^{m}\left|g\left(x_{i}\right)-g\left(x_{i-1}\right)\right| \leqslant \operatorname{Var}_{D} g(x) .
$$


Переходя в последней оценке к точной верхней грани по всем конечным подмножествам $\left\{y_{i}\right\}$ множества $E$, получаем, что $\operatorname{Var}_{E} f(y) \leqslant \operatorname{Var}_{D} g(x)$. Лемма доказана.

ПРЕДЛОЖЕНИЕ 3. Пусть $n=1, r>0, u_{0}(x) \in C((-r, r))$ и образ $u_{0}((-r, r))$ содержит интервал $(a, b)$. Предположим также, что функиия потока $\varphi(u)$ удовлетворяет условию Липшица (18) и существует непрерывное и.р. $u=$ $u(t, x)$ задачи Коши для уравнения (24) в трапеции $\Omega=\Omega_{r}=\left\{(t, x) \in \Pi_{T}\right.$ : $|x|+N t<r\}$ с начальным условием (19). Тогда производная $\varphi^{\prime}(u)$ имеет (после возможного “исправления" на множестве нулевой меры) ограниченную вариачию на любом замкнутом отрезке в $(a, b)$.

ДоказАтЕЛЬСтво. Заменив $u(t, x)$ и $u_{0}(x)$ на

$$
\min (b, \max (u(t, x), a)) \quad \text { и } \quad \min \left(b, \max \left(u_{0}(x), a\right)\right)
$$

соответственно (по теореме 1 после такой замены $u=u(t, x)$ останется непрерывным и.р. уравнения (24) с начальной функцией $\left.u_{0}(x)\right)$, будем считать, что $u_{0}(x), u(t, x) \in[a, b]$.

Пусть $E \subset(a, b)$ - множество полной меры точек Лебега функции $\varphi^{\prime}(u)$ и $[c, d] \subset(a, b)$. С учетом непрерывности функции $u_{0}(x)$ существует отрезок $J=$ $[\alpha, \beta] \subset(-r, r)$ такой, что $u_{0}(J) \supset[c, d]$. Далее, при некотором $h>0$ множество $\{(t, x): \alpha-N t \leqslant x \leqslant \beta+N t, 0<t \leqslant h\} \subset \Omega$. Покажем, что функция $f(y)=$ $y+\varphi^{\prime}\left(u_{0}(y)\right) h$ возрастает на множестве $J \cap u_{0}^{-1}(E)$. Предполагая противное, найдем значения $y_{1}, y_{2} \in(\alpha, \beta), y_{1}<y_{2}$, такие, что $u_{1}=u_{0}\left(y_{1}\right), u_{2}=u_{0}\left(y_{2}\right) \in E$ и $f\left(y_{1}\right)>f\left(y_{2}\right)$. Последнее означает, что характеристики $x_{2}(t)=y_{2}+\varphi^{\prime}\left(u_{2}\right) t$ и $x_{1}(t)=y_{1}+\varphi^{\prime}\left(u_{1}\right) t$ пересекаются при $t=t_{0}$, где

$$
t_{0}=\frac{\left[y_{2}-y_{1}\right]}{\left[\varphi^{\prime}\left(u_{1}\right)-\varphi^{\prime}\left(u_{2}\right)\right]} \in(0, h) .
$$

При этом будем считать, что функция $u_{0}(y)$ не постоянна ни в каких окрестностях точек $y_{1}, y_{2}$. Последнего всегда можно добиться, заменив $y_{1}, y_{2}$ на $y_{1}^{\prime}, y_{2}^{\prime}$, где значения $y_{1}^{\prime}, y_{2}^{\prime}$ определяются условием, что отрезки $\left[y_{1}, y_{1}^{\prime}\right],\left[y_{2}^{\prime}, y_{2}\right]$ - максимальные среди отрезков вида $\left[y_{1}, z_{1}\right],\left[z_{2}, y_{2}\right]$, на которых функция $u_{0}(y)$ принимает постоянные значения, равные $u_{1}$ и $u_{2}$ соответственно. При такой замене числитель дроби, определяющей $t_{0}$ в равенстве (39), может только уменьшиться при неизменном знаменателе. Поэтому условие пересечения характеристик $t_{0} \in(0, h)$ остается выполненным.

"Пошевелив" значения $y_{1}$ и $y_{2}$, мы можем также добиться выполнения того условия, что в любых окрестностях точек $y_{1}$ и $y_{2}$ функции $u_{0}(y)-u_{1}$ и $u_{0}(y)-u_{2}$ меняют знак. Действительно, выберем $\delta>0$ так, чтобы $\alpha<y_{1}-\delta<y_{1}+\delta<$ $y_{2}-\delta<y_{2}+\delta<\beta$ и

$$
\frac{\left[y_{2}-y_{1}+2 \delta\right]}{\left[\varphi^{\prime}\left(u_{1}\right)-\varphi^{\prime}\left(u_{2}\right)-2 \delta\right]} \in(0, h) .
$$

Так как функция $u_{0}(y)$ непрерывна и не постоянна на интервале $I=\left(y_{1}-\delta\right.$, $\left.y_{1}+\delta\right)$, то внутренность ее образа $V=\operatorname{Int} u_{0}(I)$ является невырожденным интервалом. Поскольку график $\left\{u=u_{0}(y)\right\}$ имеет нулевую меру на плоскости, то для п.в. $u \in V$ прообраз $u_{0}^{-1}(u)$ является множеством нулевой меры Лебега 
на $I$. Учитывая также, что $u_{1} \in E$ - точка Лебега функции $\varphi^{\prime}(u)$, мы можем найти значение $\bar{u}_{1} \in V \cap E$ такое, что множество $I_{0}=u_{0}^{-1}\left(\bar{u}_{1}\right)$ имеет нулевую меру и $\left|\varphi^{\prime}\left(\bar{u}_{1}\right)-\varphi^{\prime}\left(u_{1}\right)\right|<\delta$.

Далее, множества $I_{-}, I_{+} \subset I$, на которых соответственно

$$
u_{0}(y)<\bar{u}_{1} \quad \text { и } \quad u_{0}(y)>\bar{u}_{1},
$$

открыты, непусты и их замыкания покрывают $I: I=\mathrm{Cl} I_{1} \cup \mathrm{Cl} I_{2}$ (в противном случае $u_{0}(y)=\bar{u}_{1}$ на невырожденном интервале). По связности $I$ $\exists \bar{y}_{1} \in \mathrm{Cl} I_{1} \cap \mathrm{Cl} I_{2}$. Тогда $u_{0}\left(\bar{y}_{1}\right)=\bar{u}_{1}$, и поскольку $\bar{y}_{1}-$ предельная точка множеств $I_{-}, I_{+}$, то функция $u_{0}(y)-\bar{u}_{1}$ меняет знак в любой окрестности $\bar{y}_{1}$. Аналогично доказывается существование точки $\bar{y}_{2} \in\left(y_{2}-\delta, y_{2}+\delta\right)$ такой, что $\bar{u}_{2}=u_{0}\left(\bar{y}_{2}\right) \in E,\left|\varphi^{\prime}\left(\bar{u}_{2}\right)-\varphi^{\prime}\left(u_{2}\right)\right|<\delta$ и функция $u_{0}(y)-\bar{u}_{2}$ меняет знак в любой окрестности точки $\bar{y}_{2}$. Положим

$$
\bar{t}_{0}=\frac{\left[\bar{y}_{2}-\bar{y}_{1}\right]}{\left[\varphi^{\prime}\left(\bar{u}_{1}\right)-\varphi^{\prime}\left(\bar{u}_{2}\right)\right]} .
$$

Тогда с учетом (40) $\bar{t}_{0}>0$ и $\bar{t}_{0}<\left[y_{2}-y_{1}+2 \delta\right] /\left[\varphi^{\prime}\left(u_{1}\right)-\varphi^{\prime}\left(u_{2}\right)-2 \delta\right]<h$. Поэтому характеристики $x=\bar{y}_{2}+\varphi^{\prime}\left(\bar{u}_{2}\right) t$ и $x=\bar{y}_{1}+\varphi^{\prime}\left(\bar{u}_{1}\right) t$ пересекаются в точке $\left(\bar{t}_{0}, \bar{x}_{0}\right)$, где $\bar{x}_{0}=\bar{y}_{1}+\varphi^{\prime}\left(\bar{u}_{1}\right) \bar{t}_{0}$. Так как $\bar{u}_{1} \neq \bar{u}_{2}$, то одно из этих значений отлично от $u\left(\bar{t}_{0}, \bar{x}_{0}\right)$. Пусть для определенности $u\left(\bar{t}_{0}, \bar{x}_{0}\right) \neq \bar{u}_{1}$. Тогда возможны два случая: $u\left(\bar{t}_{0}, \bar{x}_{0}\right)>\bar{u}_{1}$ или $u\left(\bar{t}_{0}, \bar{x}_{0}\right)<\bar{u}_{1}$. Будем считать, что $u\left(\bar{t}_{0}, \bar{x}_{0}\right)>\bar{u}_{1}$, случай противоположного неравенства разбирается аналогично. По непрерывности $u\left(\bar{t}_{0}, x\right)>\bar{u}_{1}$ для всех $x$ из достаточно малой окрестности $W=\left(\bar{x}_{0}-\varepsilon, \bar{x}_{0}+\varepsilon\right)$ точки $\bar{x}_{0}$.

Далее, как следует из теоремы 2 , для п.в. $(t, x, v) \in \Omega \times(a, b)$

$$
\theta(v-u(t, x))=\theta\left(v-u_{0}\left(x-\varphi^{\prime}(v) t\right)\right),
$$

где функция $\theta(v)$ определена в начале доказательства теоремы 2. Поэтому указанное равенство верно на множестве общих точек Лебега левой и правой частей. При $x \in W$ значения

$$
y=y(x)=x-\varphi^{\prime}\left(\bar{u}_{1}\right) \bar{t}_{0}=x-\bar{x}_{0}+\bar{x}_{0}-\varphi^{\prime}\left(\bar{u}_{1}\right) \bar{t}_{0}=x-\bar{x}_{0}+\bar{y}_{1}
$$

"пробегают" интервал $\left(\bar{y}_{1}-\varepsilon, \bar{y}_{1}+\varepsilon\right)$, и так как функция $u_{0}(y)-\bar{u}_{1}$ меняет знак на этом интервале, $\exists x \in W: u_{0}(y(x))<\bar{u}_{1}$.

Учитывая непрерывность функций $u(t, x), u_{0}(y)$, то обстоятельство, что $\bar{u}_{1}-$ точка Лебега функции $\varphi^{\prime}(v)$, а также неравенства $u\left(\bar{t}_{0}, x\right)>\bar{u}_{1}, u_{0}(y(x))<\bar{u}_{1}$, заключаем, что $\left(t_{0}, x, \bar{u}_{1}\right)$ - точка Лебега функций

$$
\theta(v-u(t, x)) \quad \text { и } \quad \theta\left(v-u_{0}\left(x-\varphi^{\prime}(v) t\right)\right) .
$$

Поэтому

$$
0=\theta\left(\bar{u}_{1}-u\left(\bar{t}_{0}, x\right)\right)=\theta\left(\bar{u}_{1}-u_{0}\left(x-\varphi^{\prime}\left(\bar{u}_{1}\right) \bar{t}_{0}\right)\right)=1 .
$$

Получили противоречие, доказывающее, что функция $f(y)=y+\varphi^{\prime}\left(u_{0}(y)\right) h$ возрастает на множестве $J \cap u_{0}^{-1}(E)$. Поэтому $\varphi^{\prime}\left(u_{0}(y)\right)=(f(y)-y) / h$ имеет ограниченную вариацию как разность монотонных функцией. По лемме 2 $\varphi^{\prime}(u)$ является функцией ограниченной вариации на $E \cap[c, d]$ для любого отрезка $[c, d] \subset(a, b)$. В частности, для всех $u \in(a, b)$ определены односторонние 
пределы $\varphi^{\prime}(u+)=\lim _{v \rightarrow u+, v \in E} \varphi^{\prime}(v)$ и множество $\left\{u \in E: \varphi^{\prime}(u) \neq \varphi^{\prime}(u+)\right\}$ не более чем счетно. Учитывая, что $E$ - множество полной меры на $(a, b)$, получаем, что $\varphi^{\prime}(u)=\varphi^{\prime}(u+)$ п.в. на $(a, b)$; отсюда и из свойств производной липшицевой функции, в свою очередь, следует, что существует классическая производная $\varphi^{\prime}(u)=\varphi^{\prime}(u+)$ в каждой точке непрерывности функции $\varphi^{\prime}(u+)$. Для завершения доказательства остается заметить, что $\varphi^{\prime}(u+)$ является функцией ограниченной вариации на любом отрезке $[c, d] \subset(a, b)$ (уже без исключения множества нулевой меры). Предложение доказано.

ЗАмЕчАниЕ 2. Как видно из доказательства предложения 3 , функция $\varphi^{\prime}(u)$ имеет ограниченную вариацию на отрезках вида $u_{0}(J), J=[\alpha, \beta] \subset(-r, r)$.

Результат предложения 3 легко распространяется на случай произвольной области $\Omega \subset \mathbb{R}^{2}$. Справедлива

ТеОрема 6. Пусть $и(t, x)$ является непрерывным и.р. уравнения $(24)$ в области $\Omega,(a, b) \subset \operatorname{Int} u(\Omega)$, функиия потока $\varphi$ удовлетворяет условию Липшииа (18) на $(a, b)$. Тогда производная $\varphi^{\prime}$ имеет локально ограниченную вариацию на $(a, b)$.

ДокАЗАТЕЛЬство. Будем считать, что $u \neq$ const и $a<b$, случай $a=b$ тривиален. Пусть $\bar{u} \in(a, b)$. Тогда множества

$$
\Omega^{-}=\operatorname{Cl}\{(t, x) \in \Omega: u(t, x)<\bar{u}\}, \quad \Omega^{+}=\{(t, x) \in \Omega: u(t, x) \geqslant \bar{u}\}
$$

замкнуты и непусты. По связности $\Omega$ найдется точка $\left(t_{0}, x_{0}\right) \in \Omega^{-} \cap \Omega^{+}$. Тогда $u\left(t_{0}, x_{0}\right)=\bar{u}$ и любая окрестность $U$ точки $\left(t_{0}, x_{0}\right)$ содержит точки $(t, x)$, для которых $u(t, x)<\bar{u}$. Выберем окрестность вида

$$
U=\left\{(t, x):\left|x-x_{0}\right|<r-N\left|t-t_{0}\right|\right\},
$$

где величина $r>0$ достаточно мала, так что $U \subset \Omega$. Положим $v_{0}(y)=$ $u\left(t_{0}, x_{0}+y\right), y \in(-r, r)$. Тогда функции $v=v^{+}(\tau, y)=u\left(t_{0}+\tau, x_{0}+y\right)$, $v=v^{-}(\tau, y)=u\left(t_{0}-\tau, x_{0}+y\right)$ будут непрерывными и.р. уравнений $v_{\tau}+\varphi(v)_{y}=0$ и $v_{\tau}-\varphi(v)_{y}=0$ соответственно в треугольной области $\{(\tau, y): \tau>0,|y|<$ $r-N \tau\}$, удовлетворяющими начальному условию $v(0, y)=v_{0}(y)$ в смысле соотношения (19). Если $v_{0}(y) \geqslant \bar{u} \forall y \in(-r, r)$, то, как следует из теоремы 2 , $v^{ \pm}(\tau, y) \geqslant \bar{u}$, откуда $u(t, x) \geqslant \bar{u}$ в $U$, что противоречит выбору точки $\left(t_{0}, x_{0}\right)$. Итак, $\exists y \in(-r, r): v_{0}(y)<\bar{u}$, и, значит, найдутся величины $h_{1}>0, \delta \in(0, r)$ такие, что $\left[\bar{u}-h_{1}, \bar{u}\right] \subset v_{0}([-r+\delta, r-\delta])$. По предложению 3 с учетом замечания 2 обобщенная производная $\varphi^{\prime}(u)$ имеет ограниченную вариацию на $\left[\bar{u}-h_{1}, \bar{u}\right]$.

Выбирая точку $\left(t_{0}, x_{0}\right) \in \widetilde{\Omega}^{-} \cap \widetilde{\Omega}^{+}$, где замкнутые множества

$$
\widetilde{\Omega}^{-}=\{(t, x) \in \Omega: u(t, x) \leqslant \bar{u}\}, \quad \widetilde{\Omega}^{+}=\operatorname{Cl}\{(t, x) \in \Omega: u(t, x)>\bar{u}\},
$$

и используя рассуждения, аналогичные приведенным выше, получаем, что $\varphi^{\prime}(u)$ имеет ограниченную вариацию на множестве вида $\left[\bar{u}, \bar{u}+h_{2}\right], h_{2}>0$. Поэтому $\varphi^{\prime}(u)$ имеет ограниченную вариацию на объединении множеств $\left[\bar{u}-h_{1}, \bar{u}\right]$ и $\left[\bar{u}, \bar{u}+h_{2}\right]$, т.е. на отрезке $\left[\bar{u}-h_{1}, \bar{u}+h_{2}\right]$. Из произвольности $\bar{u} \in(a, b)$ следует, что $\varphi^{\prime}(u)$ имеет локально ограниченную вариацию на $(a, b)$. Теорема доказана. 
СЛЕДСТВИЕ. При выполнении условий теоремы 6 функиия потока $\varphi(u)$ дифферениируема всюду на $(a, b)$, за исключением не более чем счетного множества значений, и дважды дифберениируема почти всюду на $(a, b)$.

ДокАЗАТЕЛЬСтво. По теореме 6 можно считать, что обобщенная производная $\varphi^{\prime}(u)$ является функцией локально ограниченной вариации на $(a, b)$. Поэтому (см., например, [11]) функция $\varphi^{\prime}(u)$ непрерывна на $(a, b)$, за исключением не более чем счетного множества точек разрыва первого рода, и дифференцируема почти всюду на $(a, b)$. Отсюда непосредственно вытекает утверждение следствия.

Из теорем 4-6 выводится следующий результат.

ТеОремА 7. Пусть $и(t, x)$ является непрерывным о.р. уравнения (24) в области $\Omega,(a, b)=\operatorname{Int} u(\Omega), a \geqslant-\infty, b \leqslant+\infty$. Тогда выполнено свойство $(*)$, указанное во введении:

найдется замкнутое относительно интервала $(a, b)$ множество $F$ нулевой меры такое, что функция $\varphi$ удовлетворяет условию Липшица локально на $U=(a, b) \backslash F$ и производная $\varphi^{\prime}$ имеет локально ограниченную вариачию на $U$.

При этом $F=\varnothing$, если $\Omega$ является полосой $\left(t_{1}, t_{2}\right) \times \mathbb{R}$.

ДокАЗАТЕЛьство. По теореме $3 u(t, x)$ - и.р. уравнения $(24)$ в $\Omega$. Тогда по теореме 5 существует замкнутое относительно $(a, b)$ множество $F \subset(a, b)$ нулевой меры такое, что $\varphi(u)$ удовлетворяет условию Липшица локально на $U=$ $(a, b) \backslash F$ и, в частности, определена обобщенная производная $\varphi^{\prime}(u) \in L_{\mathrm{loc}}^{\infty}(U)$. При этом ввиду теоремы $4 F=\varnothing$, если $\Omega$ является полосой $\left(t_{1}, t_{2}\right) \times \mathbb{R}$. Пусть $\bar{u} \in U$. Выберем окрестность $(c, d) \subset U$ точки $\bar{u}$ такую, что $\varphi(u)$ удовлетворяет условию Липшица на $[c, d]$. Функция $\widetilde{u}=\max (\min (u(t, x), d), c)$ является непрерывным и.р. уравнения $(24)$ в $\Omega$ (по теореме 1 ) и удовлетворяет предположениям теоремы 6 . Тогда по теореме 6 функция $\varphi^{\prime}(u)$ имеет локально ограниченную вариацию на $(c, d)$. Из произвольности точки $\bar{u} \in U$ следует, что $\varphi^{\prime}(u)$ имеет локально ограниченную вариацию на $U$. Теорема доказана.

Мы готовы дать приложение теоремы 7 к проблеме о функциональной зависимости частных производных, изложенной во введении. Пусть $v=v(t, x)-$ $C^{1}$-гладкая функция в области $\Omega \subset \mathbb{R}^{2}$, отличная от линейной и удовлетворяющая соотношению $(1): v_{t}=\varphi\left(v_{x}\right)$ в $\Omega$, где $\varphi(u)$ - непрерывная функция. Положим $u=v_{x} \in C(\Omega)$. Дифференцируя соотношение (1) по переменной $x$ в $\mathscr{D}^{\prime}(\Omega)$, получаем, что

$$
u_{t}-\varphi(u)_{x}=0 \quad \text { в } \mathscr{D}^{\prime}(\Omega),
$$

т.е. $u=u(t, x)$ - непрерывное о.р. уравнения (41). Пусть $(a, b)=\operatorname{Int} u(\Omega)$. Тогда $a<b$ (в противном случае $v_{t}, v_{x}$ постоянны) и по теореме 7 функция $\varphi(u)$ удовлетворяет свойству $(*)$. В частности, $\varphi(u)$ дважды дифференцируема п.в. на $(a, b)$.

Этот результат дает некоторую информацию об аналитических и геометрических свойствах образов производных $C^{1}$-гладких функций двух переменных. Геометрические свойства образов производных дифференцируемых (негладких) функций в многомерном случае изучались ранее, например в работах [12], [13]. 


\section{Список литературы}

[1] Е. Ю. Панов, Обобщенные решения задачи Коши для квазилинейных законов сохранения, Дис. ... канд. физ.-матем. наук, МГУ, М., 1991.

[2] С.Н. Кружков, "Обобщенные решения задачи Коши в целом для нелинейных уравнений первого порядка", Докл. АН СССР, 187:1 (1969), 29-32.

[3] С. Н. Кружков, "Квазилинейные уравнения первого порядка со многими независимыми переменными", Матем. сб., 81:2 (1970), 228-255.

[4] Е.Ю. Панов, "K теории обобщенных энтропийных решений задачи Коши для квазилинейного уравнения первого порядка в классе локально суммируемых функций", Изв. РАН. Сер. матем., 66:6 (2002), 91-136.

[5] С.Н. Кружков, Е. Ю. Панов, "Консервативные квазилинейные законы первого порядка с бесконечной областью зависимости от начальных данных", Докл. АH CCCP, 314:1 (1990), 79-84.

[6] S. N. Kruzhkov, E. Yu. Panov, "Osgood's type conditions for uniqueness of entropy solutions to Cauchy problem for quasilinear conservation laws of the first order", Ann. Univ. Ferrara Sez. VII (N.S.), 40 (1996), 31-54.

[7] Е. Ю. Панов, "О мерозначных решениях задачи Коши для квазилинейного уравнения первого порядка", Изв. РАН. Сер. матем., 60:2 (1996), 107-148.

[8] А. Ю. Горицкий, Е. Ю. Панов, "О локально ограниченных обобщенных энтропийных решениях задачи Коши для квазилинейного уравнения первого порядка", Тр. МИАН, 236 (2002), 120-133.

[9] Г. Федерер, Геометрическая теория меры, Наука, М., 1987.

[10] М. Хирш, Дифференциальная топология, Мир, М., 1979.

[11] И. П. Натансон, Теория функиий вещественной переменной, Наука, М., 1974.

[12] J. Malý, "The Darboux property for gradients", Real Anal. Exchange, 22:1 (1996/97), $167-173$.

[13] М. В. Коробков, “Об одном обобщении теоремы Дарбу на многомерный случай”, Сиб. матем. журн., 41:1 (2000), 118-133.

M. В. Коробков (M. V. Korobkov)

Институт математики им. С. Л. Соболева СО РАН, г. Новосибирск

E-mail: korob@math.nsc.ru

\section{Е. Ю. Панов (Е. Yu. Panov)}

Новгородский государственный университет,

г. Великий Новгород

E-mail: pey@novsu.ac.ru
Поступила в редакцию

04.11 .2004 и 13.05 .2005 